\title{
Effective resolution concepts for lidar observations
}

\author{
M. Iarlori ${ }^{1}$, F. Madonna ${ }^{2}$, V. Rizi ${ }^{1}$, T. Trickl ${ }^{3}$, and A. Amodeo ${ }^{2}$ \\ ${ }^{1}$ CETEMPS/DSFC, Università Degli Studi Dell'Aquila, Via Vetoio, 67010 Coppito, L'Aquila, Italy \\ ${ }^{2}$ Consiglio Nazionale delle Ricerche - Istituto di Metodologie per l'Analisi Ambientale CNR-IMAA, \\ C.da S. Loja - Zona Industriale 85050 Tito Scalo, Potenza, Italy \\ ${ }^{3}$ Karlsruhe Institute of Technology (KIT), IMK-IFU, Garmisch-Partenkirchen, Germany
}

Correspondence to: M. Iarlori (marco.iarlori@aquila.infn.it)

Received: 31 March 2015 - Published in Atmos. Meas. Tech. Discuss.: 28 May 2015

Revised: 2 October 2015 - Accepted: 21 October 2015 - Published: 10 December 2015

\begin{abstract}
Since its establishment in 2000, EARLINET (European Aerosol Research Lidar NETwork) has provided, through its database, quantitative aerosol properties, such as aerosol backscatter and aerosol extinction coefficients, the latter only for stations able to retrieve it independently (from Raman or high-spectral-resolution lidars). These coefficients are stored in terms of vertical profiles, and the EARLINET database also includes the details of the range resolution of the vertical profiles. In fact, the algorithms used in the lidar data analysis often alter the spectral content of the data, mainly acting as low-pass filters to reduce the high-frequency noise. Data filtering is described by the digital signal processing (DSP) theory as a convolution sum: each filtered signal output at a given range is the result of a linear combination of several signal input data samples (relative to different ranges from the lidar receiver), and this could be seen as a loss of range resolution of the output signal. Low-pass filtering always introduces distortions in the lidar profile shape. Thus, both the removal of high frequency, i.e., the removal of details up to a certain spatial extension, and the spatial distortion produce a reduction of the range resolution.

This paper discusses the determination of the effective resolution (ERes) of the vertical profiles of aerosol properties retrieved from lidar data. Large attention has been dedicated to providing an assessment of the impact of low-pass filtering on the effective range resolution in the retrieval procedure.
\end{abstract}

\section{Introduction}

Smoothing and numerical derivative are typically applied in the retrieval of aerosol optical properties from lidar data, and both may act as a low-pass filter. The smoothing is a lowpass filter; instead the ideal numerical derivative enhances high frequencies (Mollova, 1999). This means that in order to perform effectively a numerical derivative of a signal, a smoothing filter has to be coupled in cascade with the ideal derivative (see Sect. 2.1). For this reason the terms "smoothing filter" and "low-pass filter" should be considered as synonymous. The smoothing is an operation frequently carried out on the raw lidar signals as well as on final products, such as the aerosol backscatter coefficient $\left(\beta_{\mathrm{a}}\right)$ (Klett, 1981; Fernald, 1984; Ansmann et al., 1992) to reduce the random noise. On the other hand, the retrieval of the aerosol extinction coefficient $\left(\alpha_{\mathrm{a}}\right)$ from a Raman lidar signal (Ansmann et al., 1992), or of the planetary boundary layer height estimation from a Rayleigh lidar signal (Matthias et al., 2004), or of the ozone profiles and the water vapor profiles with the differential absorption lidar (DIAL) technique (e.g., McGee et al., 1995; Wulfmeyer and Bösenberg, 1998), includes a numerical derivative. The application of low-pass filtering will also generate a reduction in the vertical or time resolution with respect to the unfiltered products. Moreover, often there is the need for comparing or combining different atmospheric variables and this requires that they are fully consistent in time and in space: they must be nearly co-located, coincident in time and with the same space resolution. This latter category includes, for example, the retrieval of lidar ratio $(S)$ profile or the comparison between the same quantity obtained by different instruments with different resolutions, 
like balloon-borne ozone data versus ozone lidar profiles, as pointed out by previous studies (e.g., Masci, 1999). In those cases, inconsistencies could arise if data are not compared at the same resolution. For example, to obtain an $S$ profile, a high-resolution aerosol backscatter coefficient profile, having well-resolved layers, could be combined with a heavily smoothed, low-resolution simultaneous extinction profile, where the same layers can be not well resolved. This would result in a biased estimation of the actual values of the lidar ratio (see Sect. 3 and 3.1).

In general, even if the data samples after the filtering process emerge separated by the same spatial step they had before being processed, this spatial step does not represent the effective resolution of the filtered profile. The primary aim of this paper is to study the problem of the effective resolution (ERes) estimation for lidar products using a different approach with respect to those already presented in the literature (Godin at al., 1999; Beyerle and McDermid, 1999; Shcherbakov, 2007; Trickl, 2010; Leblanc et al., 2012; Pornsawad et al., 2012). A parametric study of several smoothing filters is performed, and the results provide recommendations on the optimization of lidar signal processing.

Although it is more common to consider the vertical spatial resolution for lidar profiles, the effective resolution concept can be easily generalized and extended to the time resolution, for example, in the case of the time series of lidar profiles.

This paper is organized as follows: in Sect. 2, the foundations of smoothing and numerical derivation are summarized. Section 3 is devoted to the ERes operative estimation based on both the application of the well-known Rayleigh criterion (Born and Wolf, 1999) and the calculation of the so-called noise reduction ratio (NRR) (Orfanidis, 2010). In the Appendix A-D, different constructions of low-pass filters are introduced discussing their advantages and drawbacks. The "Summary and conclusions" section reports the outcome of the paper and includes recommendations and suggestions useful for the analysis of lidar data.

\section{Smoothing and derivation of a lidar profile: the digital filter approach}

As pointed out in previous works (Pappalardo et al., 2004; Matthias et al., 2004; Whiteman, 1999), the most used algorithms to smooth or differentiate the data within the EARLINET community are those involving some kind of sliding least-squares polynomial fitting. Adopting this point of view in a straightforward manner is not very efficient (Press et al., 2007) and "the digital filter approach and the concept of smoothing polynomials yield identical results" (Steffen, 1986). These digital filters based on smoothing polynomials are widely known as Savitzky-Golay filters (SG) (Savitzky and Golay, 1964) and include both smoothers and $n$ th-order differentiators: they will be discussed in some detail in Appendix A.

Without going into detail, as largely discussed in several books and papers on digital signal processing (DSP) (e.g., Hamming, 1998; Orfanidis, 2010), a digital filter is a numerical procedure or algorithm that transforms a given sequence of numbers $x(n)$ (the inputs signal) in another $y(n)$ (output signal) that has some more desirable properties (like less noise, for example). The general time-domain relationship between the system output value at time $n$ and the input values for a linear-time-invariant (LTI) system can be written as

$y(n)=\sum_{k=-\infty}^{\infty} h(k) x(n-k)$.

In Eq. (1), the sequence $h(k)$ is the so-called impulse response and it represents the wanted LTI transformation. Unless otherwise specified, the word "signal" refers to a generic input/output of the filter. In the case of real-time DSP, Eq. (1) cannot be used directly since the calculation of output value $y(n)$ at the current time $n$ will require future discrete-time input samples (for this reason the LTI system is required to be causal). Moreover the summation in Eq. (1) has infinite limits.

However, for the current study only finite impulse response (FIR) filter will be examined, i.e., only those filters for which the impulse response, $h(k)$, has a finite number of non-zero elements $(2 N+1, k=-N, \ldots, N)$. Furthermore, the analysis of lidar signals is typically carried out offline and, for this reason, "future" input samples are always available (Orfanidis, 2010). On the basis of the above considerations, Eq. (1) can be written as

$\left\{\begin{array}{l}y(n)=\sum_{k=-N}^{N} h(k) x(n-k), n=N, \cdots, n_{\max }-N-1 \\ n_{\max } \geq 2 N+1 .\end{array}\right.$

Equation (2) is a representation of the so-called non-causal linear-time-invariant (LTI) finite impulse response (FIR) digital filter (Orfanidis, 2010). If the impulse response consists of $M=2 N+1$ samples and if the length of the input signal $x(n)$ is $n_{\max }\left(n=0,1, \ldots, n_{\max }-1\right)$, then the length of the output signal $y(n)$ will have $M+n_{\max }-1$ samples, if we do not consider the limits for $n$ in Eq. (2). When $y(n)$ is calculated for $n$ outside these limits, i.e., for $n<N$ or $n>n_{\max }-$ $N-1$, there is always a lack of one or more of the needed input samples in $x(n)$. These missing inputs are treated as zeroes in the convolution sum, and this causes a transient effect and, hence, distortion on the smoothed signal. To remove this transient effect, $n$ have been limited in Eq. (2). Of course, this implies an overall loss of information in the output signal because of the necessary removal of $N$ points at the beginning and at the end of the output signal. Anyhow, it should be noted that there are techniques (Gorry, 1990; Khan, 1987; Leach et al., 1984; Orfanidis, 2010) that are able to 
deal with this problem. In the study of processes in the troposphere, which are the primary objective of EARLINET, these transient effects could limit the capability of retrieving planetary boundary layer information, which is already affected by the incomplete overlap between the lidar transmitted beam and the receiver field of view, if not properly corrected (Wandinger and Ansmann, 2002). Indeed, if the spatial extension of the region of incomplete overlap is not well known, the smoothing of a profile including this region might bias the retrieval, for example, of the aerosol extinction profile, $\alpha_{\mathrm{a}}$, at the lower ranges.

The impulse response of a non-causal FIR filter is a real-valued sequence (this is true also for the signals considered in this study) and its discrete time Fourier transform (DTFT) (Karam et al., 2009; Hamming, 1998; Smith, 2007):

$$
\begin{aligned}
H(\omega) & =\sum_{k=-N}^{N} h(k) e^{-j \omega k}, \omega=\frac{\Omega\left[\mathrm{rad} \mathrm{s}^{-1}\right]}{f_{\mathrm{S}}\left[\text { sample s}^{-1}\right]} \\
& =\frac{2 \pi f}{f_{\mathrm{S}}}\left[\mathrm{rad} \mathrm{sample}{ }^{-1}\right]
\end{aligned}
$$

gives the frequency response, where $\omega$ (the digital frequency) is the ratio between the analog angular frequency $\Omega$ and the sampling frequency $f_{\mathrm{S}}$, which "represents a convenient normalization of the physical frequency $f$ " (Orfanidis, 2010). In general $H(\omega)$ is a complex, continuous and periodic function of $\omega$ with period $2 \pi$. For real $h(k), H^{*}(\omega)=H(-\omega)$, and all the information contained in the frequency response are confined in the range $0 \leq \omega \leq \pi$ (Kuc, 1988). The normalized frequency, $\omega / \pi$, will be used as an independent variable, when needed, in the plots of this work (Schafer, 2011).

The FIR frequency response can be written as

$H(\omega)=A(\omega) e^{j \theta(\omega)}$,

where $A(\omega)$ is the amplitude and $\theta(\omega)$ is the phase response. A sufficient condition to have linear phase is a real and symmetric impulse response (Oppenheim and Schafer, 2009). For linear-phase FIR filters with even symmetric impulse response, the phase response is a linear function of $\omega$ that can be expressed as $\theta(\omega)=-N_{0} \omega$, where $N_{0}$ is a constant. This form of $\theta(\omega)$ simply shifts in time the different frequency components of the signal by the same constant amount, avoiding phase distortion. In this study, we consider non-causal (i.e., $N_{0}=0$ ) low-pass linear-phase FIR filters with the symmetry condition $h(k)=h(-k)$. Under this assumption, $H(\omega)$ becomes a real function (Kuc, 1988) which can take positive and negative values. However when the amplitude changes sign, the phase response shows a jump discontinuity of $\pi \mathrm{rad}$ (Kohn, 1987). Note also that for a antisymmetric non-causal impulse response there is a constant term of $\pi / 2$ rad to be considered (see Sect. 2.1) in the phase response (Oppenheim and Schafer, 2009). As an example, three different $H(\omega)$ curves are shown in Fig. 1 for an SG filter obtained using a second-degree polynomial (SG2) for different values of the parameter $N$.

$H$ is a direct representation of how a filter alters the frequency content of a signal. In lidar studies, the relevant features of a signal are generally confined in the lowerfrequency portion of the signal spectrum. In the ideal case, for low frequencies, no alterations are produced on an input signal (where $H=1$, the so-called pass-band region), while the frequencies in the stop-band region (where $H=0$ ) are completely removed. Ideal low-pass filters cannot be practically realized, and each deviation from the ideal behavior will produce artifacts in the output signal. In fact, real lowpass filters are characterized by a finite transition region between the pass band and the stop band and, at higher frequencies, $H$ could be significantly different from zero and with alternating positive and negative amplitude values (ringing or side-lobe effects). As an example, a negative value of $H$ in the stop-band region could result in particularly evident high-frequency artifacts in the output signal. For this reason, the filter design normally tries to minimize the transition region and the side-lobe effects as discussed in Appendix B, C and D.

To clarify all the above effects, we considered what happens when a low-pass filter is applied to an oscillating input signal described by the following equation:

$$
\begin{aligned}
& x_{n}=\cos \left[2 \pi\left(\frac{f_{0}}{f_{\mathrm{s}}}\right) n+\pi k\left(\frac{n}{f_{\mathrm{s}}}\right)^{2}+\varphi\right] ; f_{0}=0 ; k=\frac{2}{\pi} ; \varphi=0 \\
& \Rightarrow x_{n}=\cos \left[2\left(\frac{n}{f_{\mathrm{s}}}\right)^{2}\right]=\cos \left(2 t_{n}^{2}\right) ; t_{n}=\left(\frac{n}{f_{\mathrm{s}}}\right) ; n=0,1,2, \cdots
\end{aligned}
$$

Equation (5) is a representation of the so-called linear chirp signal (McClellan et al., 2003; https://en.wikipedia.org/wiki/ Chirp) in the discrete form ( $f_{\mathrm{S}}$ is the sampling frequency), which is useful for our purpose because its spectrum contains several frequencies, starting from the DC $(\omega=0)$ to the higher ones. The results are summarized in Fig. 2. In particular, artifacts caused by the first side lobe are quite evident, appearing as waves, poorly attenuated and inverted in sign with respect to the input signal, and located where the abscissa of the smoothed signal plot is between $\sim 3.3$ and $\sim 5.5$. For this example, an SG filter has been selected because it is one of the most used smoothing filters and also because it exhibits all the abovementioned effects resulting from the smoothing process.

Due to the large dynamic range of a lidar signal, digital filters with a different frequency response could be applied at different ranges, in order to handle properly the local values of the signal-to-noise ratio (SNR). In this way, the smoothing parameters of the filter are range dependent; therefore, the smoothed lidar returns will be characterized by a range-dependent effective resolution (see Sect. 3). The range-dependent filter parameters have a small impact on the computation time required for the lidar data analysis especially when compared to standard smoothing operated by 

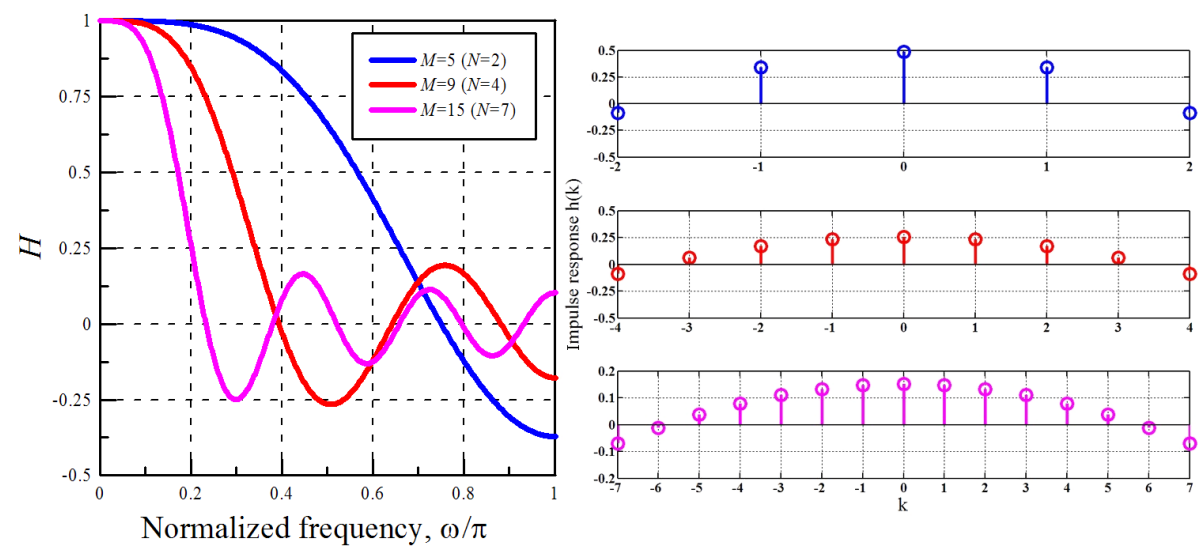

Figure 1. Left panel: frequency responses of the SG2 smoothing filter for different values of the filter length, $M=2 N+1$. Right panel: non-causal impulse responses for the different filter lengths.
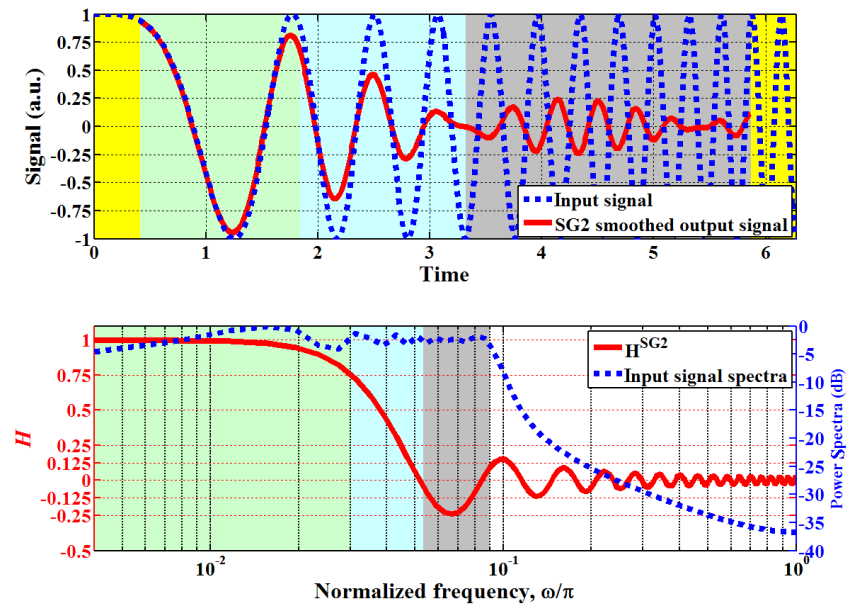

Figure 2. Upper panel: the input signal (Eq. 5) and the smoothed one using an SG2 $(N=33)$ filter. Bottom panel: the frequency response of the SG2 filter and the frequency spectra of the input signal. The signal spectral features are preserved in the light green region, where $H>0.7$ (Schafer, 2011). In the cyan area, the corresponding frequencies are sensibly damped, $0<H<0.7$. It can be clearly seen, corresponding to the gray regions in the two panels, the effect of $H<0$, which results in high-frequency artifacts in the output signal (red line in the upper panel). Note also the transient zones, marked in yellow at the boundaries of the timescale of the signal in the upper panel, where no output signal can be retrieved. $H \approx 1$ for $0<\omega / \pi<4 \times 10^{-3}$.

the least-squares polynomial fitting (see Appendix A); this is quite important in the case of centralized calculus tools like the Single Calculus Chain (SCC) (D'Amico et al., 2015), developed within EARLINET to perform a near-real time and fully automated analysis of aerosol lidar data.

In Appendix A, B, C and D, a few digital filter types that could be used in lidar data processing are described in detail. Anyhow, there are many other methods to design efficient low-pass filters (e.g., Eisele, 1998; Trickl, 2010, also used within EARLINET).

The digital filter approach enables one to calculate the random error associated with the output in a relative easy manner if compared with the covariance matrix calculations needed in the least-squares polynomial fitting (Whiteman, 1999). In fact, using the error propagation rules (Rocadenbosch et al., 2012), the variance (Var) of a filtered signal could be written as (Gans, 1992)

$\operatorname{Var}[y(n)]=\sum_{k=-N}^{N} h^{2}(k) \operatorname{Var}[x(n-k)]$.

Equation (6) is correct if no correlation exists among input signal samples and errors, which is a hypothesis frequently assumed in lidar studies and in many other scientific fields. Anyhow, if further operations are performed on a signal after the smoothing process, the error estimation must be carried out with particular care. In fact, even if measurement errors associated with samples of the input signal are uncorrelated, the errors associated with the samples of the output smoothed signal will be correlated as a consequence of the convolution process and then the covariance matrix must be applied (Gans, 1992).

\subsection{Low-pass filter and first derivative}

Besides direct smoothing, the first derivative is the other operation frequently used in lidar data analysis. The frequency response of the ideal first derivative filter is (Mollova, 1999)

$$
\begin{aligned}
& H^{(1)}(\omega)=j \omega=\omega e^{j \pi / 2}, 0 \leq \omega \leq \pi ; \\
& \left|H^{(1)}(\omega)\right|=\omega .
\end{aligned}
$$

Equation (7) shows a significant difference from a low-pass filter: since its frequency response grows linearly with $\omega$, the ideal derivative can be seen as a noise-adding process because it amplifies high frequencies, an unwanted feature for 


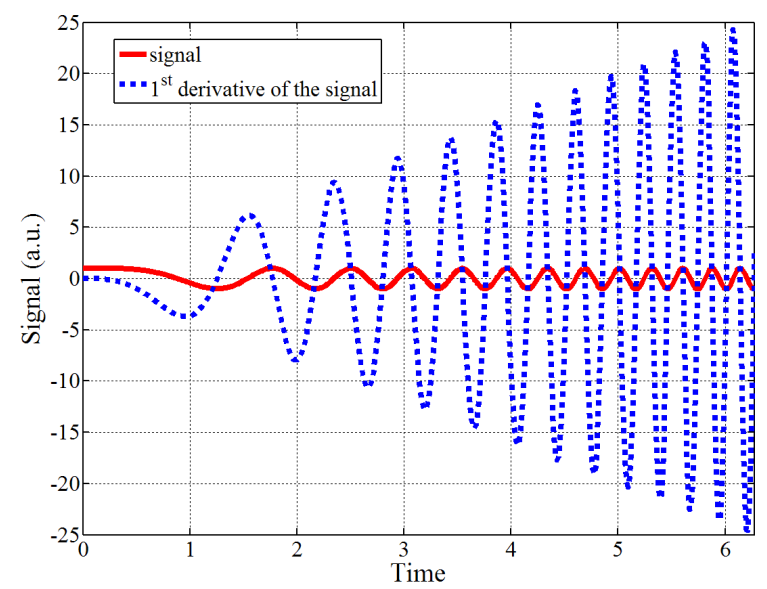

Figure 3. The signal described by Eq. (5) (red line), together with its first derivative (blue dotted line). The high-frequency components of the signal are amplified in the derivative.

our purposes. In Fig. 3, the chirp function of Eq. (5) is plotted along with its analytical first derivative; it helps to figure out why this amplification happens. In the same fashion of the example reported in Fig. 2, the portion of the differentiated signal we want to preserve is the low-frequency one (for example the part corresponding to the $[0,1]$ interval of the time axis), but the high frequencies (the noisy portion) are strongly amplified with respect to those originally included in Eq. (5); the higher the frequency, the higher the amplification, as described by Eq. (7).

Therefore a proper trade-off must be operated between the execution of the ideal derivative procedure for the whole signal and the necessary cut of the high frequencies. For this reason, the goal is to design a band-limited differentiator that, for frequencies higher than a certain cut-off value (i.e., for $\omega>\omega_{\mathrm{c}}$ ), will ideally remove the high-frequency component in Eq. (7). In other words, a low-pass differentiator is wanted, i.e., a differentiator with a frequency response like $H^{(1) \mathrm{L}}$ and that can be thought as a cascade of a low-pass filter $H^{\mathrm{L}}$ and the ideal derivative $H^{(1)}$ (Luo et al., 2005; Zuo et al., 2013):

$H^{(1) \mathrm{L}}(\omega)=H^{(1)}(\omega) H^{\mathrm{L}}(\omega)$.

The impulse response coefficients of this generic first derivative filter can be written as $h^{(1) \mathrm{L}}(k)$. This kind of impulse response is anti-symmetric $\left(h^{(1) \mathrm{L}}(k)=-h^{(1) \mathrm{L}}(-k)\right.$, $h^{(1) \mathrm{L}}(0)=0$ ) (Hamming, 1998; Smith, 2007), and a frequency response is given by Eq. (3) and Euler formulas (Wang, 2012, 2013):

$H^{(1) \mathrm{L}}(\omega)=-j \sum_{k=-N}^{N} h^{(1) \mathrm{L}}(k) \sin (\omega k)$,

where the cosine terms vanished. It is worth recalling that for low-pass filters, the terms that disappear in Eq. (3) are the sine terms, because of the even symmetry $(h(k)=h(-k))$ of

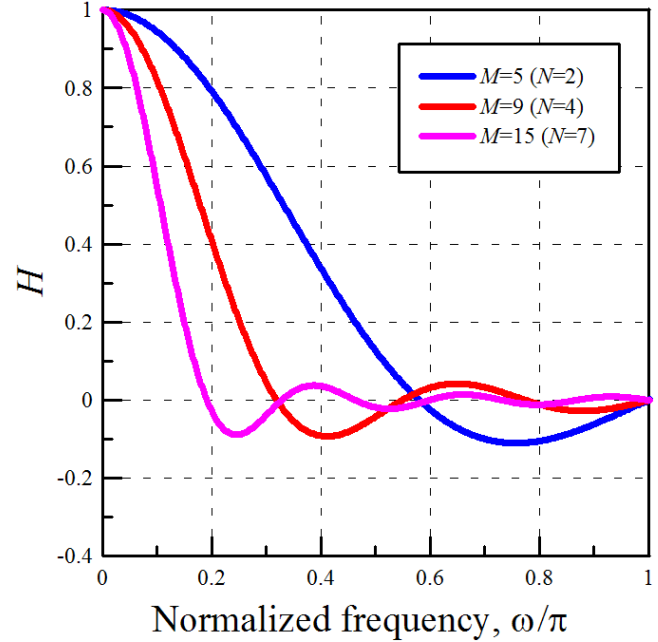

Figure 4. The frequency responses of the low-pass filter embedded with an SG2 derivative filter and estimated with Eq. (10) $\left(H^{\mathrm{L}}\right.$, i.e., the dSG2 low-pass filter) at different $M$ values.

their impulse responses. Thus, from the Eqs. (7), (8), and (9), $H^{\mathrm{L}}(\omega)$ can be written as

$H^{\mathrm{L}}(\omega)=\frac{H^{(1) \mathrm{L}}(\omega)}{j \omega}=\frac{-\sum_{k=-N}^{N} h^{(1) \mathrm{L}}(k) \sin (\omega k)}{\omega}$.

In Fig. 4, results from Eq. (10) are plotted in the case of an SG2 derivative filter. Therefore, the SG derivative filter can be understood as a cascaded system of two filters: the first one being a low-pass filter acting on the raw input signal and the second one being the ideal differentiator $(j \omega)$ and acting on the low-pass filtered signal. Prefix "d" stands for the lowpass filter prototype (i.e., dSG) associated with this first stage of the SG differentiator (inherently low pass), e.g., dSG2 for those in Fig. 4.

\section{The effective resolution}

The investigation of the synthetic lidar data inversion (Pappalardo et al., 2004) in Fig. 5 helps to recognize the effective resolution (ERes) as relevant in lidar data analysis. It highlights that the effective resolution plays an important role in assessing properly the problems that could arise when data with different resolutions are combined. In this latter figure, the aerosol layer included in the true profile at $1.4-1.6 \mathrm{~km}$ becomes heavily smoothed by the low-pass filter used in the retrieval (a dSG2 in this case). If the aerosol backscatter profile, $\beta_{\mathrm{a}}$, is smoothed, the resulting lidar ratio profile is consistent with the true one (see Fig. 5, central and right panels), both in value and in behavior. On the contrary, if aerosol backscatter profile, $\beta_{\mathrm{a}}$, is not smoothed, the lidar-ratio profile in the layer becomes quite different from the synthetic one. Outside the layer the differences between the retrieved lidar-ratio profiles 


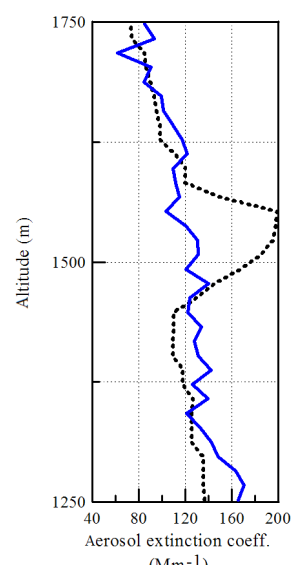

$\left(\mathrm{Mm}^{-1}\right)$

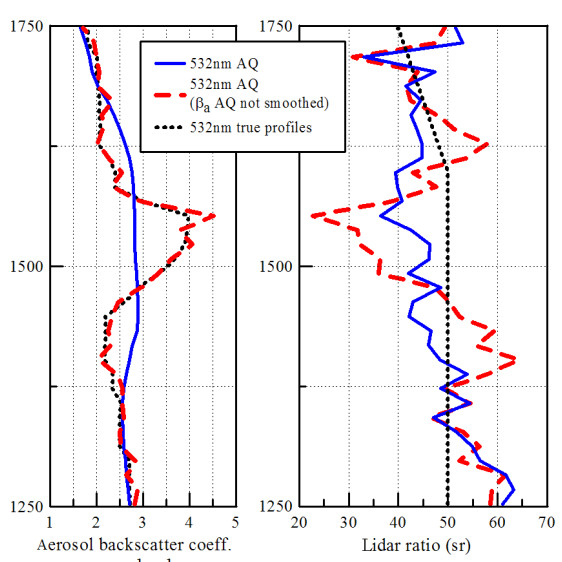

$\left(\mathrm{Mm}^{-1} \mathrm{sr}^{-1}\right)$
Figure 5. Aerosol retrievals using synthetic lidar profiles at L'Aquila (Pappalardo et al., 2004). The aerosol layer at $1.4-1.6 \mathrm{~km}$ almost disappears when smoothed by the low-pass filter used in the retrieval algorithm (blue lines). The lidar-ratio profile in the layer is not too far from the truth (black dotted lines); the same agreement is not reached if the aerosol backscatter coefficient profile, $\beta_{\mathrm{a}}$, is not smoothed (red dashed lines).

are less relevant because the aerosol field is nearly constant and for this reason less sensitive to the distortion effect of smoothing filter (Ziegler, 1981).

Two approaches will be considered for the quantitative assessment of the ERes:

- The first one is related to the distortion induced by the smoothing process on any non-trivial input signal (Enke and Nieman, 1976; Ziegler, 1981). In fact, the area preservation property (common to all the considered smoothing filters, for example see the properties of SG filters in Appendix A) implies that if the peak of a layer is reduced, its spatial width will increase and potentially could overlap with another feature present in a profile. The final result will be that it is no longer possible to distinguish one peak from another (i.e., they are no longer resolved): this means that a low-pass filter reduces the vertical resolution. This latter statement naturally leads to the use of the Rayleigh criterion (Born and Wolf, 1999) for the determination the effective resolution.

- The second approach is based on the removal of high frequencies due to the smoothing operation (Gans and Gill, 1983; Orfanidis, 2010). Since high frequencies in space domain correspond to relatively small-scale details in the lidar profiles, if they are lost in a certain amount this will imply a reduction of the resolution in the output profile with respect to the input one. Incidentally, it should be noted that since a smoothing filter damps effectively only high frequencies and since it is common to deal with white noise, the low-frequency portion of the noise is still present in the smoothed signal, for example in the form of long-wave ripples (Gans,
1992). Moreover, a link is established between the ERes estimated with each of those two approaches and the corresponding cut-off frequency definition, as discussed in Sect 3.4 (Godin et al., 1999; Masci, 1999; Beyerle and McDermid, 1999; Leblanc et al., 2012).

Before discussing the two abovementioned methods, using the results of Sect. 2.1, an answer will be provided to the question about how to obtain a lidar-ratio profile that comes from aerosol extinction and backscatter profiles with the same effective resolution.

\subsection{Obtaining profiles with the same effective resolution: the lidar ratio case}

To retrieve the aerosol extinction profile, $\alpha_{\mathrm{a}}$ (Ansmann, 1992), a first-derivative filter is applied. The frequency response of the embedded low-pass filter $\left(H^{\mathrm{L}}\right)$ can be found from Eq. (10), or more directly if a Gaussian derivative filter is employed (see Appendix D for details on Gaussian filters). The calculation of $H^{\mathrm{L}}$ gives the solution to the problem of retrieving a consistent lidar ratio and without a hypothesis or assessment about the effective resolution itself of the profiles involved: they only need the same resolution. In fact, once $H^{\mathrm{L}}$ is known, because the properties of the convolution and of the Fourier transform (Rabiner et al., 1970; Rabiner and Gold, 1975; Kuc, 1988; Oppenheim and Schafer, 2009), with the aid of fast Fourier transform (FFT) algorithms it is possible to smooth the corresponding aerosol backscatter profile, $\beta_{\mathrm{a}}$, with this filter. In this way both the profiles are with the same effective resolution, since their frequency spectrum has been changed using the same low-pass filter. To illustrate better the above concepts, in Fig. 6, a retrieval of the optical parameters is performed starting from simulated elastic/Raman lidar data (Ansmann, 1992; Pappalardo et al., 2004) with an aerosol layer $1000 \mathrm{~m}$ thick. The signals have been simulated for the Rayleigh signal at $351 \mathrm{~m}$ and for the corresponding nitrogen Raman signal at $382 \mathrm{~m}$, without adding noise or background. Both the SG2 derivative and the Gaussian derivative filters are employed to retrieve the aerosol extinction profile. Then the corresponding embedded low-pass filters are used to smooth the raw-resolution aerosol backscatter profile. Both the extinction and the backscatter coefficient profiles with the same ERes are combined to get an estimation of the lidar ratio. Figure 6 shows that, besides the good results for the retrieval of the lidar ratio inside the actual simulated layer, an accurate result is also obtained in the zone immediately outside the layer, i.e., where the filter distorts the profiles with respect to the true layer. In Fig. 6 it is also shown that wrong lidar-ratio values are obtained in almost all the aerosol layers if the aerosol backscatter profile, $\beta_{\mathrm{a}}$, is smoothed with a different low-pass filter $\left(H\right.$ instead of $\left.H^{\mathrm{L}}\right)$. 

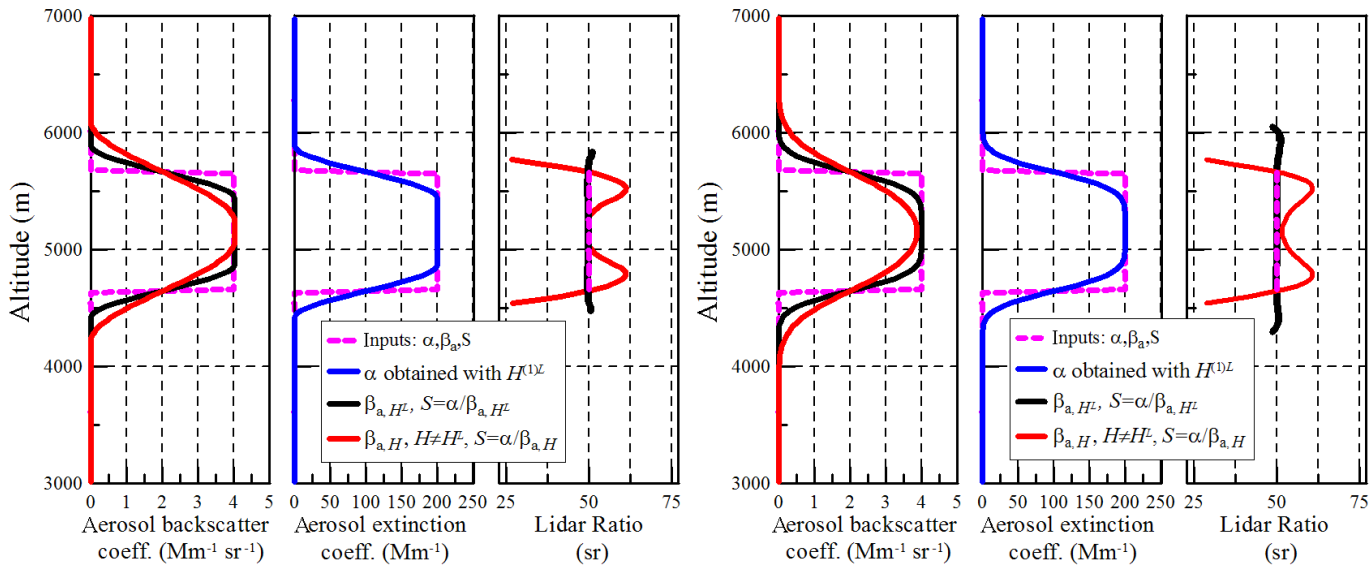

Figure 6. In the left plots, the aerosol extinction profile, $\alpha$ (blue line), has been retrieved by means of an SG2 derivative filter with $N=$ 7 (frequency response is indicated with $H^{(1) \mathrm{L}}$ ). The aerosol backscatter profile, $\beta_{\mathrm{a}, H^{\mathrm{L}}}$ (black line), is obtained by smoothing the one retrieved at raw resolution (not shown) with the correct low-pass filter: the dSG2 filter with $N=7$ (i.e., the smoothing filter $H^{\mathrm{L}}$ ). The other aerosol backscatter profile, $\beta_{\mathrm{a}, H}$ (red line), is retrieved using a low-pass filter of the same family (dSG2), but with $N=14$ : then $H \neq H^{\mathrm{L}}$. The right plots show the same vertical profiles, but using the Gaussian filters. The parameter driving the Gaussian derivative filter is $\sigma$. In this case the value used to get the aerosol extinction profile (blue line), $\alpha(\sigma=4)$, it is the same needed in the Gaussian filter to smooth the raw-resolution backscatter profile (black line). A Gaussian filter with a doubled $\sigma$ value $(\sigma=8)$ is used to obtain the other aerosol backscatter profile, $\beta_{\mathrm{a}, H}$ (red line). In all the plots the magenta lines indicate the input simulated profiles.

\subsection{The effective resolution: the Rayleigh criterion}

The Rayleigh criterion is generally accepted in spectroscopy for the determination of the minimum resolvable detail (Born and Wolf, 1999). It is an empirical criterion and states that two peaks are considered fully resolved if the drop in intensity between them is lower than $74 \%$ of the peak intensity. This is a result of the diffraction formulation that says that the imaging process is named diffraction-limited when the first diffraction minimum of the image of one source point coincides with the maximum of another. The application of Rayleigh criterion to the determination of the effective resolution could be done by analyzing the effect of a low-pass filter on a pair of unitary pulses. Operatively, two unitary pulses at fixed distance are smoothed by a low-pass filter whose parameters are changed to increase the signal distortion. By increasing $N$ for SG filters with fixed $P$, or $\sigma$ for Gaussian filters, it is possible to find the maximum value of the filter parameter that still allows resolving two smoothed pulses according to the Rayleigh criterion. For SG filters, after $P$ is changed, the whole procedure is repeated again to evaluate the results of applying the criterion to different polynomial degrees. Then, the effective resolution associated with the smoothing filter considered is exactly this distance. Moreover, this procedure, also known as the "step function" method, has been already tested in the frame of the first EARLINET algorithm intercomparison (Pappalardo et al., 2004).

There are alternative approaches to estimate the ERes such as the analysis of the smoothing effect on the full width at half maximum (FWHM) of a finite impulse (Leblanc et al., 2012), the response to a Heaviside step function (VDI -
Verein Deutscher Ingenieure, 1999; Eisele and Trickl, 2005; Vogelmann and Trickl, 2008, Trickl, 2010), or the application of the regularization theory to lidar data processing (Pornsawad et al., 2012; Shcherbakov, 2007). However, for SG filters, the "step function" method apparently shows some ambiguous results as can be seen in the examples reported in Fig. 7. In fact with SG filters (with $P \geq 2$ ) it can be difficult to properly define whether the Rayleigh criterion is satisfied (or not) because of the occurrence of anomalous features like bumps between the two smoothed peaks (Fig. 7 central panel) and/or of the displacement of the smoothed peaks with respect to their unsmoothed position (Fig. 7, lower panel). In these cases, the ratio used for the application of the Rayleigh criterion is evaluated between the intensity at the peak and the intensity at the midpoint between the two peaks. Moreover, because the intensity at the midpoint is not always the absolute minimum, this ratio provides a more conservative estimation of the ERes. The drawbacks in the application of the Rayleigh criterion could represent a further problem caused by the presence of side lobes in the frequency response of the SG filters. For the Gaussian filter and for filters whose side lobes are of limited amplitude (as the SG2 + Blackman window (SG2 + Blk), see Appendix C) in comparison with SG filters, these problems are no longer observed.

However, the "step function" method used in the case of the SG0 filter (also known as the boxcar or the moving average, see Appendix A) of length $M$ leads to a first operative definition for the ERes. In fact, from Fig. 8, it should be clear that the effective resolution in this case is simply reduced by a factor of $M=2 N+1$, because under the action of the SG0 

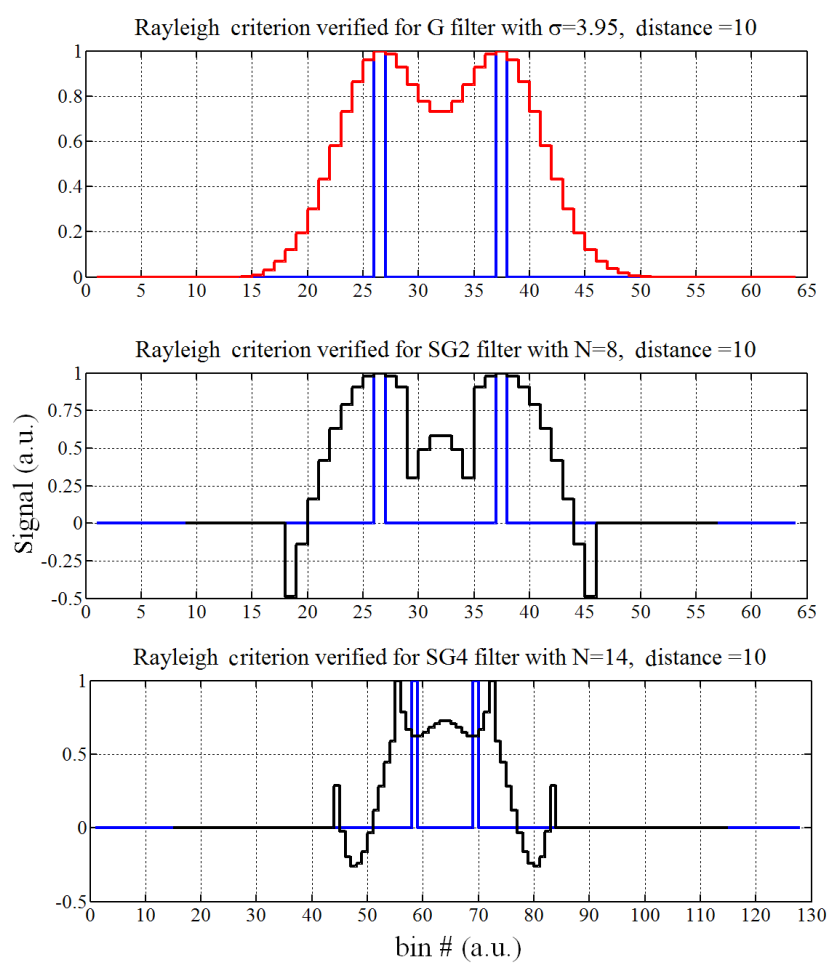

Figure 7. The Rayleigh criterion applied to a couple of one bin thick $\left(\Delta R_{\text {raw }}=1\right.$, in arbitrary units, a.u.) unitary pulses (blue line) distant 10 bins. The lower and central panels show (black lines) the smoothed profile obtained using the SG filters: unwanted features (under-shots, the central "bump" and the displacement of the smoothed peaks) are quite evident in both cases. The profile obtained with the Gaussian filter (upper panel, red line) has no problems. All smoothed profiles are normalized to the maximum.

all the involved data points will be equally weighted. So the ERes ( $\left.\Delta R_{\text {eff }}\right)$ associated with the boxcar filter can be explicitly written as

$\left.\Delta R_{\mathrm{eff}}^{\mathrm{SG} 0}\right|_{\text {Ray }}=(2 N+1) \Delta R_{\text {raw }}$.

It is worth mentioning that for the SG0 the effective resolution is also equal to the inverse of its impulse response coefficient (multiplied by the raw resolution $\Delta R_{\text {raw }}$ ) which, for any given $N$, is a constant independent of $k$,

$h^{\mathrm{SG} 0}(k)=\frac{1}{2 N+1}$.

The use of cascade filters can be exploited to solve the observed ambiguity in the application of the Rayleigh criterion for SG filters with $P \geq 2$. In fact, the cascaded filters, constructed with our simple rule (stated in Appendix B), make plausible that a L1 filter has almost the same ERes of the cascade L1 - L2 filter. For example, the ERes estimated for the SG2 - SG4 cascade should approximate the value of the ERes of the SG2 filter. Moreover, Fig. 9 shows that the application of the Rayleigh criterion to the SG2 - SG4 cascade does not

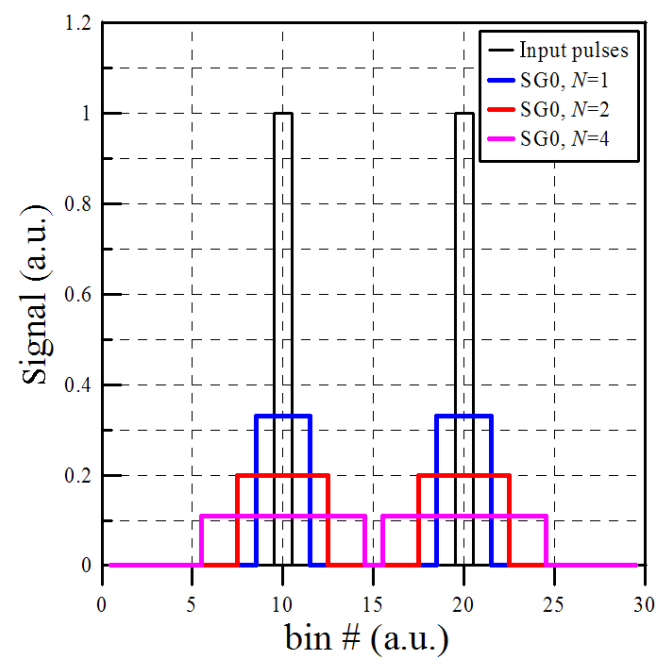

Figure 8. Two unitary pulses (thickness $=\Delta R_{\text {raw }}=1$, distance between pulses $=9$ bins, black line) after the application of SG0 with $N=1$ (length $M=2 N+1=3$, blue line) and $N=2$ (length $M=2 N+1=5$, red line), become $3 \Delta R_{\text {raw }}{ }^{-}$and $5 \Delta R_{\text {raw }}$-wide pulses respectively. For $N>4$ the two pulses cannot be distinguished: $\left.\Delta R_{\mathrm{eff}}^{\mathrm{SG} 0}\right|_{\text {Ray }}=9$ bins [a.u.].

show the central bump problem. Figure 10 (left panel), which shows the behavior of the ERes of several SG filters and their corresponding "equivalent" cascaded filters, evidences that the occurrence of the anomalous "bump" feature in Fig. 7, has a limited effect in the determination of the ERes $(<5-$ $10 \%$ difference) for these filters.

Exploiting the quite evident linear relationship between the ERes and $N$ shown in Fig. 10, the following linear relationship can be obtained:

$$
\left\{\begin{array}{l}
\left.\Delta R_{\mathrm{eff}}^{\mathrm{SG} 2 \cdot \mathrm{SG} 4}\right|_{\text {Ray }}=(1.17 N-0.09) \Delta R_{\mathrm{raw}} \\
\left.\Delta R_{\mathrm{eff}}^{\mathrm{SG}}\right|_{\text {Ray }}=(1.24 N-0.24) \Delta R_{\mathrm{raw}} \\
\left.\Delta R_{\mathrm{eff}}^{\mathrm{SG} 4 \cdot \mathrm{SG} 6}\right|_{\text {Ray }}=(0.80 N-0.65) \Delta R_{\mathrm{raw}} \\
\left.\Delta R_{\mathrm{eff}}^{\mathrm{SG} 4}\right|_{\text {Ray }}=(0.74 N-0.48) \Delta R_{\mathrm{raw}} \\
\Delta R_{\mathrm{eff}}^{\mathrm{SG} 6 \cdot \mathrm{SG} 8 \text { Ray }}=(0.60 N-0.78) \Delta R_{\mathrm{raw}} \\
\left.\Delta R_{\mathrm{eff}}^{\mathrm{SG} 6}\right|_{\text {Ray }}=(0.62 N-0.86) \Delta R_{\mathrm{raw}}
\end{array}\right.
$$

For the other filters under investigation (dSG2, SG2 + Blk, and Gaussian), the application of the Rayleigh criterion does not give particular problems. Thus, Fig. 10 (right panel) shows that for these filters the ERes can also be written using the linear relationships,

$$
\left\{\begin{array}{l}
\left.\Delta R_{\mathrm{eff}}^{\mathrm{dSG} 2}\right|_{\text {Ray }}=(1.55 N+0.83) \Delta R_{\mathrm{raw}} ; \\
\left.\Delta R_{\mathrm{eff}}^{\mathrm{SG} 2+\mathrm{Blk}}\right|_{\text {Ray }}=(0.80 N+0.20) \Delta R_{\mathrm{raw}} \\
\left.\Delta R_{\mathrm{eff}}^{G}\right|_{\text {Ray }}=(2.79 \sigma-1.04) \Delta R_{\text {raw }}
\end{array}\right.
$$

For example, if an aerosol extinction profile is retrieved from a nitrogen Raman lidar signal with a raw resolution of $\Delta R_{\text {raw }}=15 \mathrm{~m}$ by using an SG2 derivative filter (i.e., the lowpass filter to consider is the dSG2) with $N=30$, its estimated 


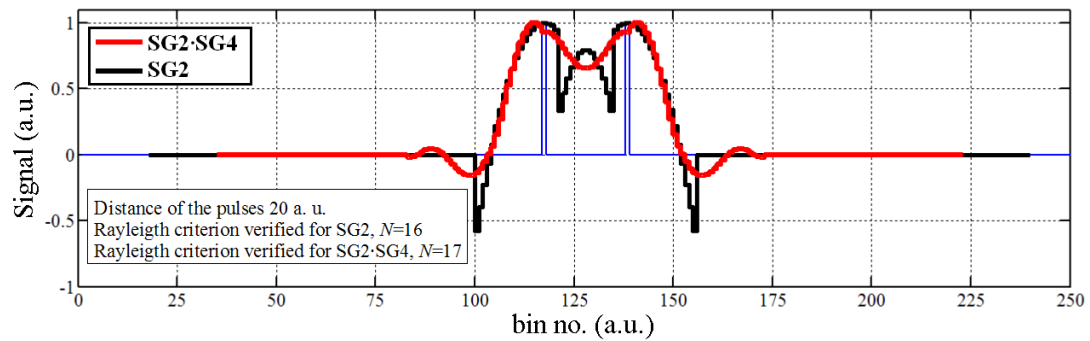

Figure 9. The Rayleigh criterion applied to 2 unitary pulses ( 1 bin thick and at a distance of 20 bins) in the case of the SG2 filter (black line) and of the SG2 - SG4 filter (red line). The cascaded filter does not exhibit the appearance of central "bump". The $N$ values in legend indicate the maximum possible value to resolve the two smoothed pulses.
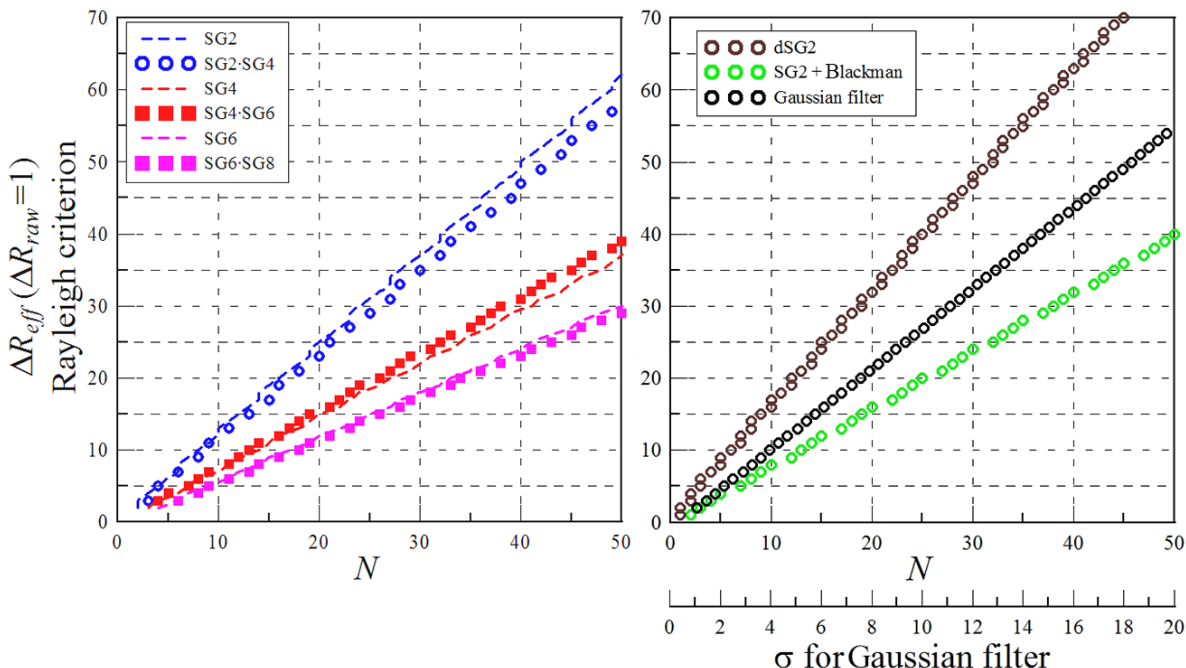

Figure 10. Left panel: the ERes estimated with the Rayleigh criterion applied to SG filters up to order $P=6$ as a function of $N$. For all the polynomial orders examined $(2,4,6)$, small differences $(\leq 5-10 \%)$ in the ERes are found between the results obtained applying the criterion on SG filter and the corresponding "equivalent" cascade filters. Right panel: the ERes obtained for three different low-pass filters (dSG2, SG2 + Blk, Gaussian) for which the application of Rayleigh criterion does not show anomalous features.

ERes will be of $700 \mathrm{~m}$. Because of the constraints on $N$ and $\sigma$ discussed in Appendix A and D, the ERes obtained with Eqs. (13) and (14) will always be positive and larger than the raw resolution, $\Delta R_{\text {raw }}$, and lower than $(2 N+1) \Delta R_{\text {raw }}$ (upper limit given by the SG0). The linear fit is performed with the constraint that SG filters with $N=P / 2$ do not smooth and, therefore, do not change the vertical resolution (i.e., $\left.\Delta R_{\text {eff }}=\Delta R_{\text {raw }}\right)$. As an example of how these constraints are applied for the SG4 filter, the condition $N=P / 2 \mathrm{im}$ plies that with $N=2$ the filter will not cause any smoothing and, hence, $\Delta R_{\text {eff }}=\Delta R_{\text {raw }}$ (=1 in our plots). Then, to obtain the fitting parameters, the linear relationship has been constrained through to the point $\left(N=2, \Delta R_{\mathrm{eff}}=1\right)$. Regardless of whether the linear fit is constrained or not, the estimated slope does not significantly change while the estimated intercept point has low impact on the ERes determination (up to $\pm 1 \cdot \Delta R_{\text {raw }}$, a value that can be interpreted as indetermination on the estimation of the ERes). As $N$ or $\sigma$ increase in Eqs. (13) and (14), the weight of the intercept point in the de- termination of the ERes becomes less and less relevant. The numerical difference in the ERes calculated with or without the intercept point for the SG2 is negligible for any value of $N$; for the SG6 the difference becomes $<10 \%$ for $N>15$, and for the Gaussian filter it is lower than $10 \%$ when $\sigma>4.5$.

Although the use of the Rayleigh criterion leads to simple and ready-to-use linear relationships for the calculation of the ERes, no universal equation encompassing all low-pass filters has been found. In fact, for any given new smoothing filter, the whole procedure has to be repeated from scratch.

\subsection{The effective resolution: the NRR criterion and the SNR matching criterion}

Removal of the noise embedded in a signal is the main purpose in the application of a low-pass filter. The amount of zero-mean white noise removed by a generic filter has already been explicitly assessed in literature (Gans and Gill, 1983; Brown, 2000). Thus, the ratio between the in- 
put ( $\operatorname{Var}_{\text {IN }}$ ) and the output (VaroUT) noise variances can be taken as a measure of the noise removed from an input signal after the filter smoothing. This quantity is also called noise reduction ratio (NRR) and depends only on the impulse response of the filter under investigation (see Chapter 8.3 and Appendix A.2 in Orfanidis, 2010; Mitra, 2001; Gans and Gill, 1983). Formally,

$\mathrm{NRR}=\frac{\operatorname{Var}_{\mathrm{OUT}}}{\operatorname{Var}_{\mathrm{IN}}}=\sum_{k=-N}^{N}[h(k)]^{2}$.

By using the explicit formula for the ERes associated with the SG0 filter and Eqs. (11), (12), and (15),

$$
\left\{\begin{array}{l}
\mathrm{NRR}^{\mathrm{SG} 0(\mathrm{~N})}=\sum_{k=-N}^{N}\left[h^{\mathrm{SG} 0}(k)\right]^{2}=\frac{1}{2 N+1} ; \\
\left.\Delta R_{\mathrm{eff}}^{\mathrm{SG} 0(\mathrm{~N})}\right|_{\mathrm{NRR}}=(2 N+1) \Delta R_{\text {raw }}=\frac{\Delta R_{\text {raw }}}{\mathrm{NRR}^{\mathrm{SG}(\mathrm{N})}} .
\end{array}\right.
$$

Equation (16) enables a general hypothesis on how to estimate the effective resolution by means of the NRR. In fact, it can be inferred that the ERes associated with a generic low-pass filter $L(\hat{p})$, characterized by a set of parameters, $\hat{p}=\left(p_{1}, p_{2} \cdots, p_{n}\right)$ (e.g., $\hat{p}=(N, P)$ for SG-based filters or $\hat{p}=(\sigma)$ for a Gaussian filter $)$, can be written by means of the general equation

$\left.\Delta R_{\mathrm{eff}}^{\mathrm{L}(\hat{p})}\right|_{\mathrm{NRR}}=\frac{\Delta R_{\mathrm{raw}}}{\operatorname{NRR}^{\mathrm{L}(\hat{p})}}$.

In other words, it is possible to say that since low-pass filtering alters the SNR, it is reasonable to assume that if a signal shows the same SNR after the smoothing with different lowpass filters, then those filters act on the signal in a similar fashion: they cause the same reduction of resolution. Therefore, the output profiles will have the same ERes. To implement operatively these concepts, the SG0 filter for different values of $N$ is applied on a generic signal, and then the corresponding SNR of the smoothed signal is calculated. Applying a generic low-pass filter $L(\hat{p})$ on the same signal, an optimization process can be performed to find the values of $\left[N_{0}, \hat{p}_{0}\right]$ that reduces the average difference between the two SNRs to zero, as close as possible (SNR matching criterion). Mathematically,

$$
\begin{aligned}
& \overline{\Delta \mathrm{SNR}_{N, \hat{p}}}=\overline{\mathrm{SNR}^{\mathrm{SG}(\mathrm{N})}-\mathrm{SNR}^{\mathrm{L}(\hat{p})}} \\
& \Rightarrow\left[N_{0}, \hat{p}_{0}\right]: \overline{\Delta \mathrm{SNR}_{N_{0}, \hat{p}_{0}}} \approx 0 .
\end{aligned}
$$

Finally, by using Eq. (16), the ERes of a $L\left(\hat{p}_{0}\right)$ smoothing filter becomes

$\left.\Delta R_{\text {eff }}^{\mathrm{L}\left(\hat{p}_{0}\right)}\right|_{\mathrm{SNRm}}=\left(2 N_{0}+1\right) \Delta R_{\text {raw }}$

In Fig. 11, an example of the similar SNRs achievable by using two different types of low-pass filters is shown. The results of the ERes obtained using the Eqs. (17), (18), and (19)

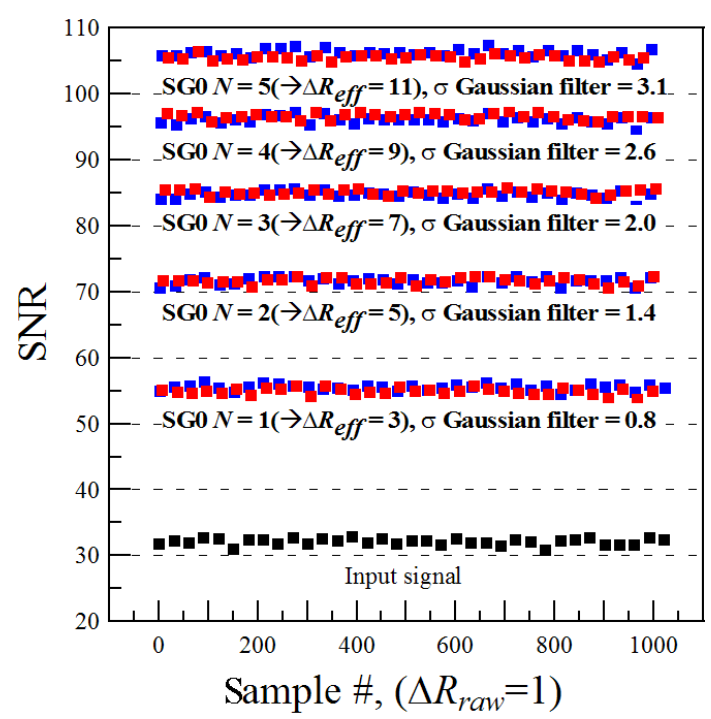

Figure 11. The input SNR of a test signal is shown with the blacksquares line. The SNRs after applying an SG0 smoothing filter with different values of $N$ are shown with blue-squares lines. The SNRs of the signal smoothed using a Gaussian filter with a $\sigma$ that satisfies Eq. (18) are also drawn with red-squares lines. Note the good agreement between the blue-squares and red-squares lines.

for several other low-pass filters are reported in Fig. 12. The analysis of Fig. 12 provides a clear confirmation of the equivalence between the NRR criterion and the SNR matching criterion. For this reason Eq. (17) provides an easy way to estimate the ERes for all types of smoothing filters, instead of using the less general (and more time-consuming) SNR matching procedure (Eqs. 18 and 19). In fact, with the NRR criterion, the estimate of the effective resolution is based only on the impulse response of the smoothing filter employed, which is known or it can easily be calculated. Figure 12 also shows that the ERes calculated with the NRR criterion can be expressed by using linear relationships, as for the Rayleigh criterion in Sect. 3.2. The linear regressions obtained from the NRR criterion for the same type of smoothing filters as the ones discussed in Sect. 3.2 are

$$
\left\{\begin{array}{l}
\left.\Delta R_{\mathrm{eff}}^{\mathrm{SG} 2 \cdot \mathrm{SG} 4}\right|_{\mathrm{NRR}}=(0.98 N+0.30) \Delta R_{\mathrm{raw}} \\
\left.\Delta R_{\mathrm{eff}}^{\mathrm{SG}}\right|_{\mathrm{NRR}}=(0.89 N+0.11) \Delta R_{\mathrm{raw}} ; \\
\left.\Delta R_{\mathrm{eff}}^{\mathrm{dS} 2}\right|_{\mathrm{NRR}}=(1.61 N+1.25) \Delta R_{\mathrm{raw}} ; \\
\left.\Delta R_{\mathrm{eff}}^{\mathrm{SG}}\right|_{\mathrm{NRR}}=(0.57 N-0.15) \Delta R_{\mathrm{raw}} ; \\
\left.\Delta R_{\mathrm{eff}}^{\mathrm{SG} 2+\mathrm{Blk}}\right|_{\mathrm{NRR}}=(0.96 N+0.04) \Delta R_{\mathrm{raw}} \\
\left.\Delta R_{\mathrm{eff}}^{\mathrm{SG} 6}\right|_{\mathrm{NRR}}=(0.42 N-0.27) \Delta R_{\mathrm{raw}} ; \\
\left.\Delta R_{\mathrm{eff}}^{G}\right|_{\mathrm{NRR}}=(3.53 \sigma+0.02) \Delta R_{\mathrm{raw}}
\end{array}\right.
$$

As can be seen from Eqs. (13), (14), and (20), comparison of the ERes estimated using the Rayleigh criterion and NRR criterion reveals differences as well as similarities. A convenient way to summarize the results obtained for both criteria and to understand their main differences is to study the behavior of the frequency responses of the smoothing fil- 

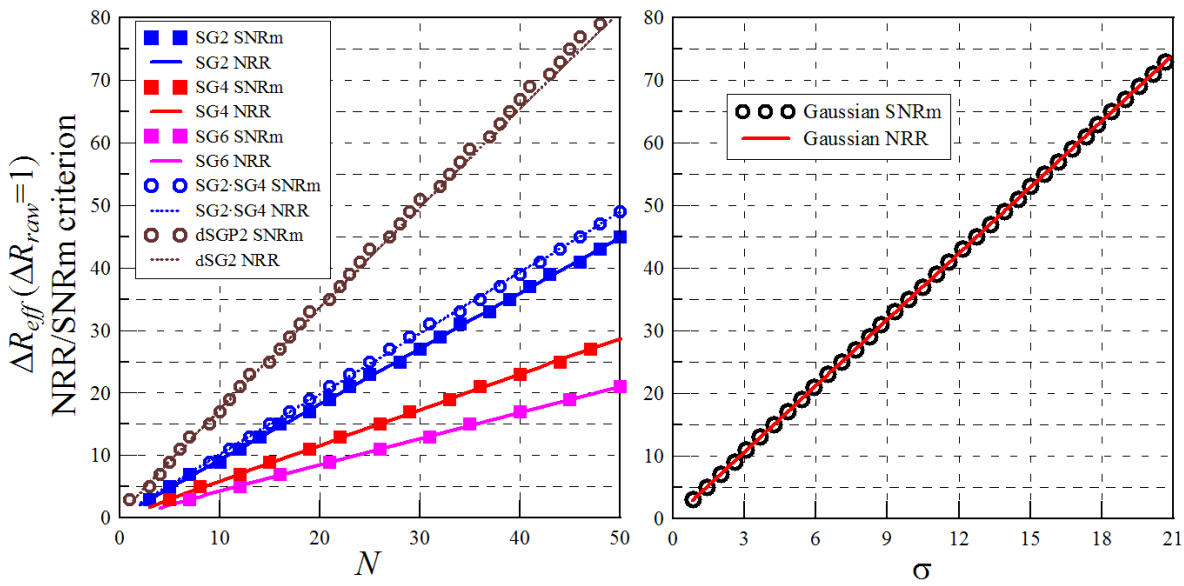

Figure 12. Left panel: the ERes estimated as a function of $N$ with the NRR criterion (solid lines) and with the SNR matching criterion (SNRm, dotted lines) are shown for different SG smoothing filters. The ERes values for the SG2 + Blk filter are not shown and they are between the SG2 and the SG2 - SG4 curves. Right panel: the ERes using the Gaussian filter with different $\sigma$. Note that the NRR and SNRm criteria give almost the same results.

ters (Fig. 13). With the NRR criterion, the same value of the ERes is obtained when the frequency responses have approximately the same pass band (i.e., for $\omega / \pi<4 \times 10^{-2}$ in the upper plot of Fig. 13), which is the region between $\omega=0$ and the canonical definition of the cut-off frequency, $\omega_{\mathrm{c}}=\left.\omega\right|_{H_{-3 \mathrm{~dB}}}=\left.\omega\right|_{H \approx 0.7}$.

On the contrary when, using the Rayleigh criterion, a common value of the ERes is obtained when the frequency responses share almost the same stop-band extension, irrespective of significant differences that may occur in the pass band. The stop-band frequency, $\omega_{\mathrm{sb}}$, is defined as the frequency corresponding to the first zero of $H(\omega)$ (Schafer, 2011) for filters having side lobes. For the Gaussian filter and the SG2windowed filter, i.e., in case of frequency responses with both a significantly wide transition band and without side lobes of relevant amplitude (see Appendix C, Fig. C1 and Fig. D1), the stop-band frequency can be taken as $\left.\omega\right|_{H=0.1}$, the upper limit of the transition band (Cappellini and Emiliani, 1986). Given the above definitions, the stop-band frequencies for the bottom plot of Fig. 13 are close to each other, since for all the filters they are comprised between $\omega / \pi \approx 7 \times 10^{-2}$ and $\omega / \pi \approx 9 \times 10^{-2}$.

As discussed so far, smoothing filters always convey a distortion action on signals and a quantification of this effect is highly desirable. In fact, as shown by use of the NRR criterion, the smoothing of a signal could not always lead to significant improvement in the SNR (this is due to the saturation effect achieved when almost all the noise is removed). For example, in Fig. 5 the layer structure is lost by the distortion action of the applied low-pass filter. For this reason, in a smoothing operation it seems very relevant to find the limit over which the (undesirable) distortion of an underlying input signal could become more relevant than the concurrent (desirable) decrease of the noise level predicted by
Eq. (15). Previous papers related to spectroscopic studies actually found this limit analyzing SG filters (Enke and Nieman, 1976; Ziegler, 1981; Gans and Gill, 1983; Rzhevskii and Mardilovich, 1994). As a potential follow-up to this paper, it would be interesting to apply the outcome of this spectroscopic studies to the lidar signals with the aim to optimize the ERes retrieval and, more in general, the whole lidar signal processing.

\subsection{The effective resolution: the cut-off frequency}

The considerations emerging from the analysis of Fig. 13 allow linking both the approaches provided for the ERes estimation to the cut-off frequency. The cut-off frequency associated with the frequency response of a smoothing filter can be used for estimating the effective resolution (Godin, 1987; Godin et al., 1999; Masci, 1999; Beyerle and McDermid, 1999; Leblanc et al., 2012). For this reason, considering a generic low-pass filter $L(\hat{p})$, the following equation is valid:

$\Delta R_{\mathrm{eff}}^{L(\hat{p})}=\pi \frac{\Delta R_{\mathrm{raw}}}{\omega_{\mathrm{c}}}$.

Of course, the definition of effective resolution in Eq. (21) depends on the value chosen for $\omega_{\mathrm{c}}$ and, therefore, on the actual pass-band (or bandwidth) definition. Figure 13 suggests that the most suitable value of $\omega_{\mathrm{c}}$ depends on the chosen criterion for the ERes estimation. In order to find this value from Eq. (21),

$\omega_{\mathrm{c}}=\pi \frac{\Delta R_{\mathrm{raw}}}{\Delta R_{\mathrm{eff}}^{L(\hat{p})}}$.

In this way, once the ERes is evaluated for a given low-pass filter $L(\hat{p})$ with a given criterion, Eq. (22) allows estimating the value of its frequency response at $\omega=\omega_{\mathrm{c}}$. The $\pi$ 

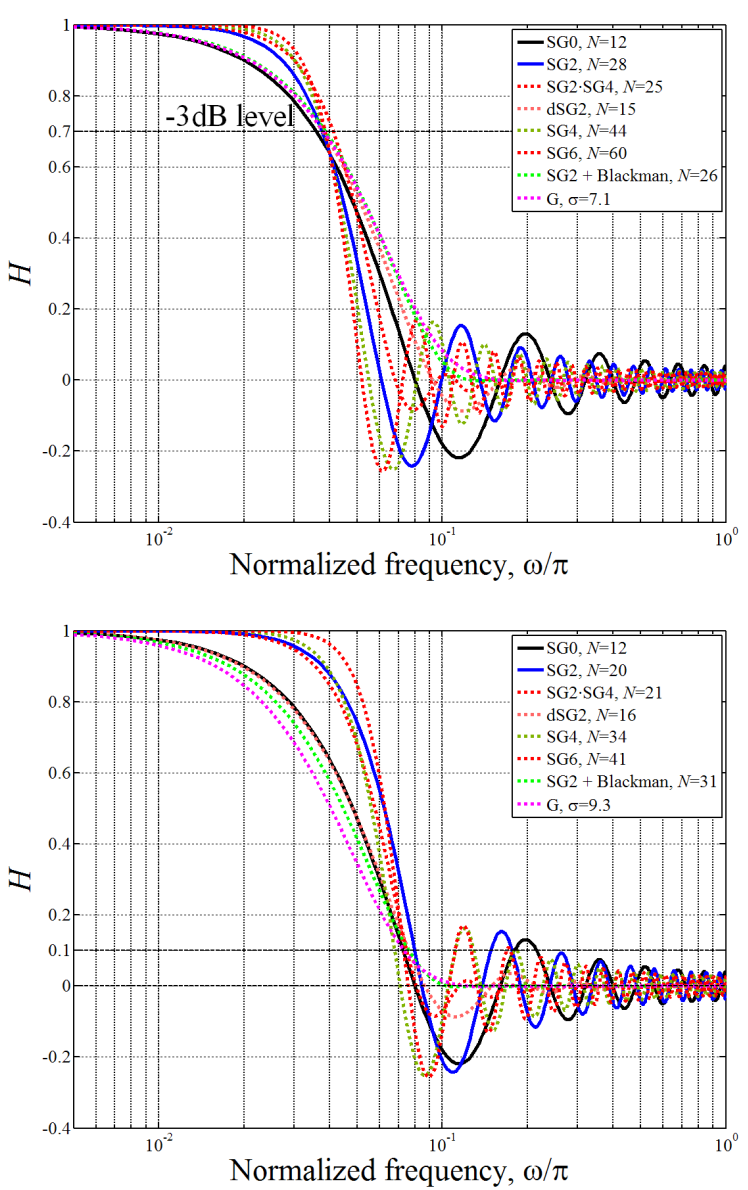

Figure 13. Upper panel: frequency responses of different low-pass filters with the same ERes $\left(=25 \Delta R_{\text {raw }}\right)$ estimated using the NRR criterion. The curves show similar behavior at lower frequencies and have nearly the same cut-off frequency $\omega_{\mathrm{c}}=\left.\omega\right|_{H_{-3 \mathrm{~dB}}}$. Bottom panel: the same curves as in the upper panel, but with the ERes $\left(=25 \Delta R_{\text {raw }}\right)$ calculated using the Rayleigh criterion. In this latter case, the common feature of the frequency responses curves is the extension of the stop-band region. $H \approx 1$ for $0<\omega / \pi<4 \times 10^{-3}$ for all the curves.

multiplication factor is necessary to obtain $\Delta R_{\text {eff }}=\Delta R_{\text {raw }}$ (i.e., no smoothing) for $\omega_{\mathrm{c}}=\pi$. For example, with the NRR criterion, the cascade $\mathrm{SG} 2 \cdot \mathrm{SG} 4$ with $N=25$ will provide a value of $\Delta R_{\text {eff }} \approx 25$ a.u. $\left(\Delta R_{\text {raw }}=1\right.$, from Eq. 17 or 20$)$, which implies a $\omega_{\mathrm{c}} / \pi \approx 0.04$; thus, once estimated at the cut-off value, the frequency response of the selected filter gives $H(0.04) \approx 0.72$ (Fig. 14, right panel). Indeed, from Fig. 14, as far as the NRR criterion is considered, the values of the frequency responses at $\omega=\omega_{\mathrm{c}}$ given by Eq. (22) for any given $\Delta R_{\text {eff }}$ and for any smoothing filter (or at least within those analyzed) are quite constant and they range within $\sim 0.65$ and $\sim 0.72$. Therefore, using the NRR criterion and Eq. (21), for a given low-pass filter $L(\hat{p})$, the cutoff frequency defined as $\left.\omega_{\mathrm{c}}^{\mathrm{L}(\hat{p})}\right|_{\mathrm{NRR}}=\left.\omega\right|_{H_{-3 \mathrm{~B}}^{\mathrm{L}(\hat{p})}}=\left.\omega\right|_{H^{\mathrm{L}(\hat{p})} \approx 0.7}$ appears as the best value to be chosen to estimate the ERes.
For the Rayleigh approach (Fig. 15), the ERes calculated using Eq. (21) is similar to those estimated using Eqs. (13) and (14) if the cut-off frequency of the low-pass filter $L(\hat{p})$ is $\left.\omega_{\mathrm{c}}^{\mathrm{L}(\hat{p})}\right|_{\mathrm{Ray}}=\omega_{\mathrm{sb}}^{\mathrm{L}(\hat{p})} / 2$ (Orfanidis, 2010). To summarize, Eq. (21) can be rewritten for both the NRR and the Rayleigh criterion as

$$
\left\{\begin{array}{l}
\left.\Delta R_{\mathrm{eff}}^{\mathrm{L}(\hat{p})}\right|_{\mathrm{NRR}} \approx \pi \frac{\Delta R_{\text {raw }}}{\left.\omega_{\mathrm{c}}^{\mathrm{L}(\hat{p})}\right|_{\mathrm{NRR}}} \approx \pi \frac{\Delta R_{\text {raw }}}{\omega\left(H_{-3 \mathrm{~dB}}^{\mathrm{L}(\hat{p})}\right)} \\
\left.\Delta R_{\mathrm{eff}}^{\mathrm{L}(\hat{p})}\right|_{\text {Ray }} \approx \pi \frac{\Delta R_{\text {raw }}}{\left.\omega_{\mathrm{c}}^{\mathrm{L}(\hat{p})}\right|_{\text {Ray }}} \approx \pi \frac{2 \Delta R_{\text {raw }}}{\omega_{\mathrm{sb}}^{\mathrm{L}(\hat{p})}} .
\end{array}\right.
$$

Equation (23) provides a general frame for the consideration done in Sect. 3.3 and related to Fig. 13. Furthermore, the second formula in Eq. (23) provides a kind of general equation, or at least a rule of thumb, also for the ERes retrieval based on the use of Rayleigh criterion. Instead the first equation is not really needed because the general equation for the NRR criterion is Eq. (17). It is important to mention that Eq. (23) has been obtained using the low-pass filters studied in this work.

\section{Summary and conclusions}

Noise removal in the retrieval of lidar optical properties using low-pass filters implies the removal of a certain amount of the useful information contained in the signal. Smoothing procedures affect both the magnitude and the vertical extension of the features contained in a profile. Moreover, the likely presence of several separated layers (of aerosol, ozone, etc.) in a lidar profile raises the question of whether they are well resolved or not after they have been smoothed. Therefore, it is important to introduce the definition of effective resolution (ERes) associated with a lidar profile to take into account the smoothing effects. The digital filter approach to investigate the smoothing effects provides several advantages with respect to the standard least-squares approach like

- faster algorithms able to properly deal with the large dynamic range of a lidar signal, which is an interesting feature concerning the automatic data processing chain like the SCC (D'Amico et al., 2015);

- easier statistical error analysis (Rocadenbosch et al., 2012);

- ready-to-use effective resolution definitions through an analysis of the impulse/frequency response;

- methods to design the most efficient low-pass filters to satisfy specific needs in the lidar signal processing.

In this work, a parametric study of several smoothing filters has been presented also with the aim to provide recommendations in support of the selection of the filter type for the aerosol lidar applications. The estimation of the ERes does 

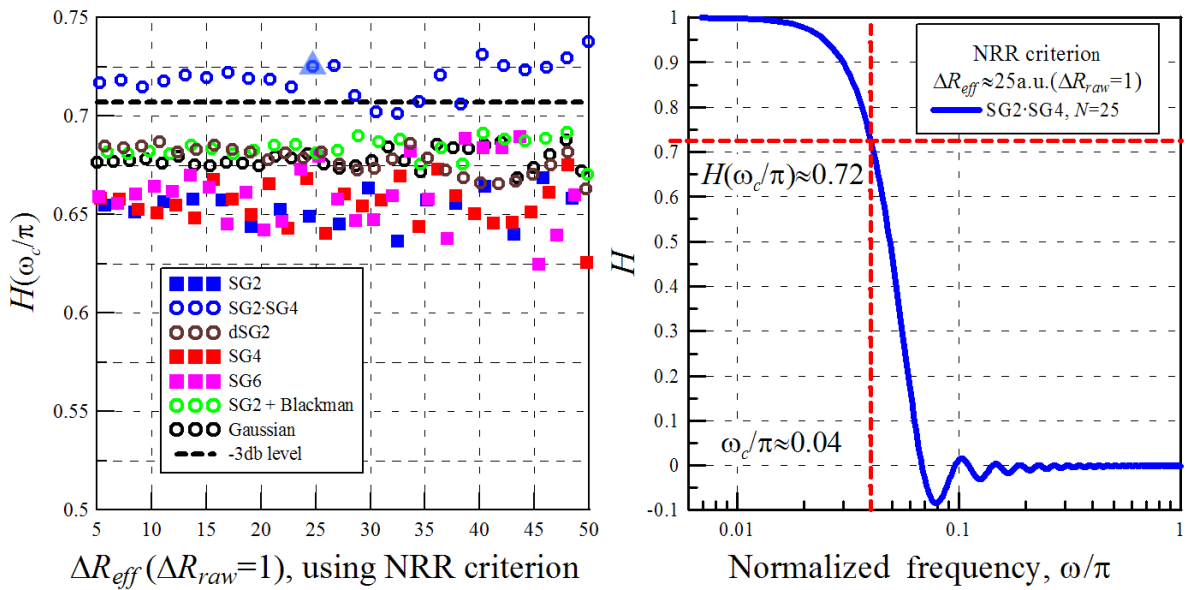

Figure 14. Left plot: the value of the frequency responses function at $\omega_{\mathrm{c}} / \pi$ plotted as a function of the effective resolution (Eq. 22, NRR criterion), for all the examined low-pass filter. Right plot: a sketch of the procedure to estimate the single value of the frequency response at $\omega_{\mathrm{c}} / \pi$, in the case of the point indicated by the blue triangle in the left plot: according to Eq. (22), the value of $\omega_{\mathrm{c}} / \pi$ is about 0.04 and the corresponding $H(0.04)$ is $\approx 0.72$.

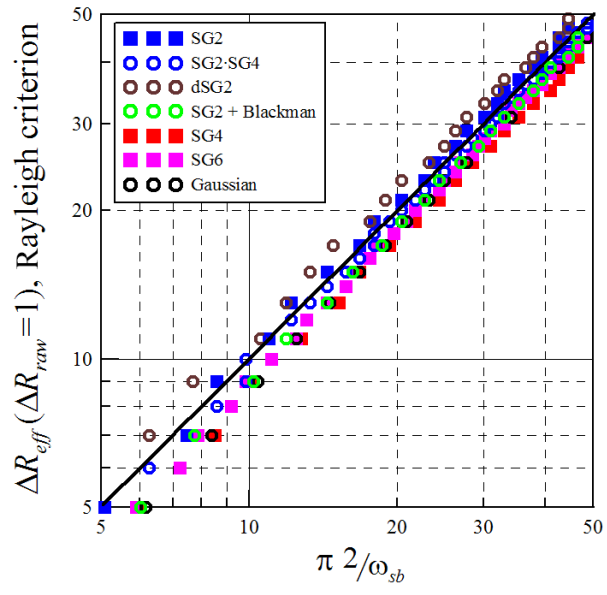

Figure 15. For all the investigated smoothing filters, the ERes obtained with the Rayleigh criterion as function of the corresponding value of the quantity $\pi \cdot 2 / \omega_{\mathrm{sb}}^{\mathrm{L}(\hat{p})}$. The identity line is drawn in black.

not provide any ultimate indication on the best filter to be applied in the of lidar data analysis. This is because the ERes does not contain any information about the spectral behavior of the different filters. Among the investigated filters, for example the cascaded Savitzky-Golay filters are well suited for the lidar data analysis. This class of filters is characterized by

- the preservation of the spectral features of the signal in the pass band;

- a narrow frequency transition band;

- reduction of the side-lobe effects;

- a larger transient zone in time domain.
The Gaussian filter are characterized by

- efficient suppression of noise;

- less accurate preservation of the signal in the frequency pass band;

- a large frequency transition band.

Moreover, as long as the choice is between the SG2 derivative filter and the Gaussian derivative filter, the employment of the latter seems recommendable when the first derivative of a signal is required.

The advantages of the DSP approach are used, for example, to solve practical problems of lidar studies, i.e., how to calculate the lidar ratio ensuring that both the retrieved aerosol extinction and aerosol backscatter profiles have the same resolution. Thus, an operative ERes estimation method has been presented by taking into account

- the Rayleigh criterion, which assesses the spatial resolution and hence our ability to resolve (or not) close aerosol layers;

- the NRR criterion, which assesses the amount of noise reduction associated with a specific low-pass filter design.

Both criteria can also be considered as a measure of the loss of information on the vertical scale of the signal. The NRR criterion underlines that smoothing filters are able to remove efficiently only the high-frequency noise. In fact, the presence of low-frequency noise remains almost unchanged and will continue to affect the lidar retrievals. Comparison between these two criteria allows the identification of a simple linear relationship between the effective resolution and the filter parameters. For the same filter with same parameters, 
using different criteria, the ERes shows differences. The discrepancies are limited to a maximum of about $30 \%$ in case of SG or Gaussian filter, while for other filters they are less significant $(<20 \%)$ or not relevant $(<5 \%$ for $\mathrm{dSG} 2$ case). The analysis of the filter's frequency responses shows that

- similar effective resolutions are obtained with the Rayleigh criterion for those filters that share a comparable stop band $\left(\omega_{\mathrm{sb}}\right)$, irrespective of their behavior in the pass band;

- similar effective resolutions are obtained with the NRR criterion for different filters that have similar values in the $-3 \mathrm{~dB}$ cut-off frequency, $\left.\omega\right|_{H_{-3 \mathrm{~dB}}}$, i.e., a comparable pass band.

Though feasible for any filter type, the ERes estimation based on the Rayleigh criterion shows some drawbacks and appears more elaborated and less general with respect to the use of the NRR criterion. In fact, a ready-to-use equation to estimate the effective resolution has found the NRR criterion applicable to all the smoothing filters. For this reason the NRR approach to the ERes estimation would appear the most suitable in terms of providing a generalized application. Nevertheless, the results of the analysis done in Sect. 3.4, allow one to obtain also for the Rayleigh criterion a specific equation for the ERes estimation based on the knowledge of $\omega_{\mathrm{sb}}$.
Finally it is worth mentioning that, within the lidar community, other approaches rooted in the numerical derivative problem have been proved to be effective, and there are other methods providing alternative and reasonable ERes definitions (Leblanc et al., 2012; Pornsawad et al., 2012; Trickl, 2010; Shcherbakov, 2007; Eisele and Trickl, 2005; VDI Verein Deutscher Ingenieure, 1999). For this reason a more exhaustive comparison with other approaches to the evaluation of the ERes will be likely done in future in the frame of EARLINET activities. 


\section{Appendix A: The Savitzky-Golay filter}

The SG approach is faster and relatively easier to implement than standard least-squares calculations, though, according to the theory, they produce the same results (Savitzky and Golay, 1964). In fact, the coefficients $h(k)$ in Eq. (2) have to be calculated just once for fixed filter parameters. For SG filters this means fixed $N$ (i.e., the filter's half-length; $M=$ $2 N+1$ is the filter's length) and fixed polynomial degree, $P$. In contrast, when using the standard least-squares smoothing, a new and complete calculation of polynomial coefficients has to be done for each point of the signal, even if $N$ and $P$ are fixed (Press et al., 2007). The minimum $N$ required to perform a significant smoothing is related to the polynomial degree through the relation $2 N>P$ (Schafer, 2011), and for $2 N=P$ there are no differences between the input and the output signals (no smoothing). With SG filters, in principle, a variable $N$ could be used when smoothing or differentiating a signal as well as a different $P$ if required (Barak, 1995), and the computation time is still shorter than for the least-squares algorithm.

The SG filters are popular in many scientific fields because of the following:

- They preserve all the first $P+1$ moments (with $P=$ $0,2,4$, i.e., $P_{\text {even }}$, because for fixed $N$, the smoothed signal will not change if either $P_{\text {even }}$ or $P_{\text {odd }}=P_{\text {even }}+1$ is used). This property is connected to the flat frequency response in the pass band as reported in Fig. 2. This feature enables quite faithful preservation of the lowfrequency component of the signal (Karam et al., 2009). For example, the moving/sliding average, which is the zeroth order SG filter (SG0), does preserve both the area (its zeroth-order moment) below a main peak in the signal (e.g., an aerosol layer) and the mean position (the first-order moment) of a symmetric peak, but does not preserve the standard deviation (the secondorder moment), which is an estimation of the peak width (Ziegler, 1981). In order to preserve higher-order moments (Bromba and Ziegler, 1981), the signal should be smoothed using an SG filter with a higher-degree polynomial $P$.

- The SG filter pass band depends on both $N$ and $P$ as can be seen in Eq. (B2). A higher $P$ tends to increase the filter's pass band, but this translates to worse performances in terms of noise removal, while a higher $N$ has an opposite effect (see Figs. 1 and 4). This suggests a trade-off between higher pass-band extension (i.e., less signal distortion) and better noise removal (Orfanidis, 2010; Savitzky and Golay, 1964; Press et al., 2007; Turton, 1992).

- The impulse response of the SG $n$ th-order derivative filter, for given $P$ and $N$, can be directly calculated (Savitzky and Golay, 1964). This is another useful property of the Savitzky-Golay method. The way the SG filters are constructed implies that low-pass filtering is an embedded feature of the derivative. It should be noted that the SG first-order derivative filter will produce the same result on a signal for $P_{\text {odd }}$ and the next even degree $P_{\text {odd }}+1$ (for $P=1$ and $P=2$, for $P=3$ and $P=4$, etc.) for a fixed $N$. The degree of flatness in the pass band associated with the corresponding low-pass filter (see Sect. 2.1) has to be assessed accordingly (Luo et al., 2005).

- The transition band (see Fig. C1) of an SG filter is generally smaller than that of other filters with similar pass band (see Figs. C1 and D1). This is a valuable characteristic (Schafer, 2011).

- The presence of side lobes is the main problem with the SG filters. As a consequence the output signal is contaminated with high-frequency artifacts as seen in Fig. 2. Moreover, the amplitude of these side lobes is significantly higher with respect to other low-pass filters, as can clearly be seen by comparing Figs. 1, 4, and D1. In fact, for SG2 filters the observed amplitude for the peak of the first side lobe is about -0.25 , which implies a signal suppression of only $75 \%$, so SG filters do not offer a great performance in the stop-band region. The attenuation value for the first side lobe does not significantly change for the examined SG filters; it becomes just slightly worse when $P$ increases (Schafer, 2011).

\section{Appendix B: Cascaded filters}

One of the methods that enable the reduction of the side lobes in the frequency response of a low-pass filters is the cascade technique. By taking advantage of commutative and associative properties of the convolution operation (D'Antona and Ferrero, 2006) and because convolution in time-domain equals multiplication in frequency domain (Das and Chakraborty, 2012), two or more low-pass filters can easily be applied in cascade. The behavior in frequency domain of cascaded filters is the product of their single transfer functions, a property used in Eq. (8). Thus, for a cascade of filters the resulting impulse and the frequency responses can be written, respectively, as

$\left\{\begin{array}{l}h^{\mathrm{L}_{\mathrm{C}}}(k)=h^{\mathrm{L} 1}(k) * h^{\mathrm{L} 2}(k) * \ldots * h^{\mathrm{Ln}}(k) ; \\ H^{\mathrm{L}_{\mathrm{C}}}(\omega)=H^{\mathrm{L} 1}(\omega) \cdot H^{\mathrm{L} 2}(\omega) \ldots \cdot H^{\mathrm{Ln}}(\omega) .\end{array}\right.$

Cascading two (or more) identical low-pass filters will effectively damp the amplitude of the side lobes. However, a drawback of cascading identical filters is the reduction of the pass-band extension. The "equivalent" cascaded filter is the one that preserves the pass-band characteristic of the "single" filter (L1) but with much less pronounced side lobes. 

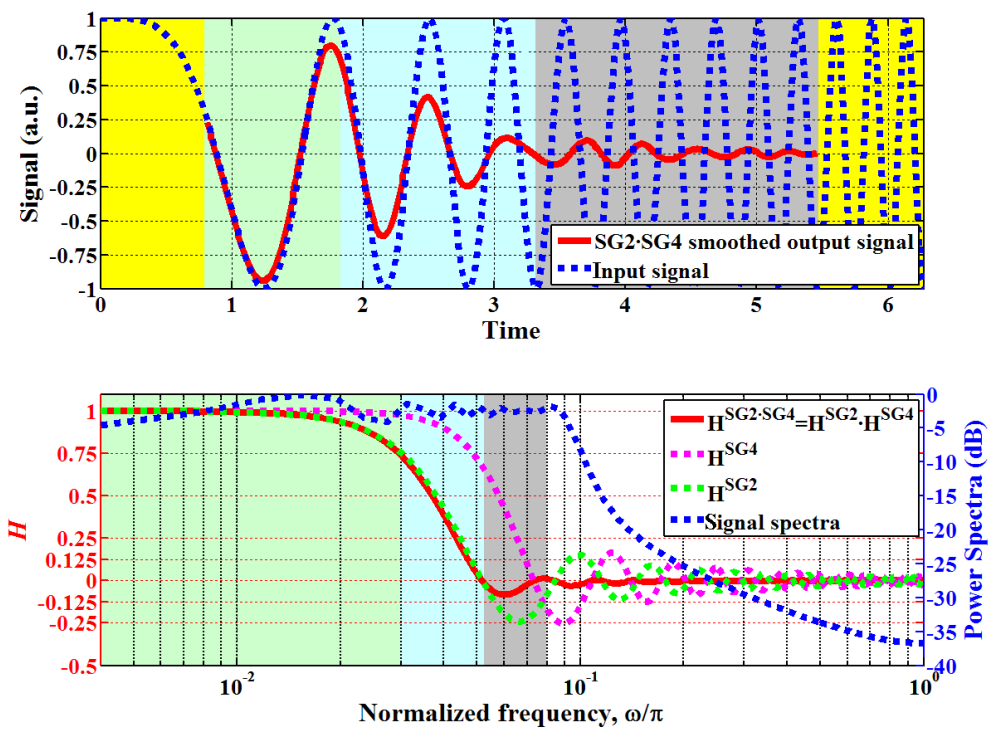

Figure B1. Upper plot: the chirp signal (blue dashed line) is smoothed by SG2 and an SG4 in cascade (red line), both with the same $N=33$. A comparison in time domain with Fig. 2 evidenced the larger transient region (in yellow) with respect to the "single" SG2, $N=33$.The cascaded filter frequency response (bottom plot) exhibits a pass band similar to the SG2 but with reduced side lobes. This latter feature virtually eliminates high-frequency artifacts in the smoothed signal in upper panel. Color code like Fig. $2, H \approx 1$ for $0<\omega / \pi<4 \times 10^{-3}$.
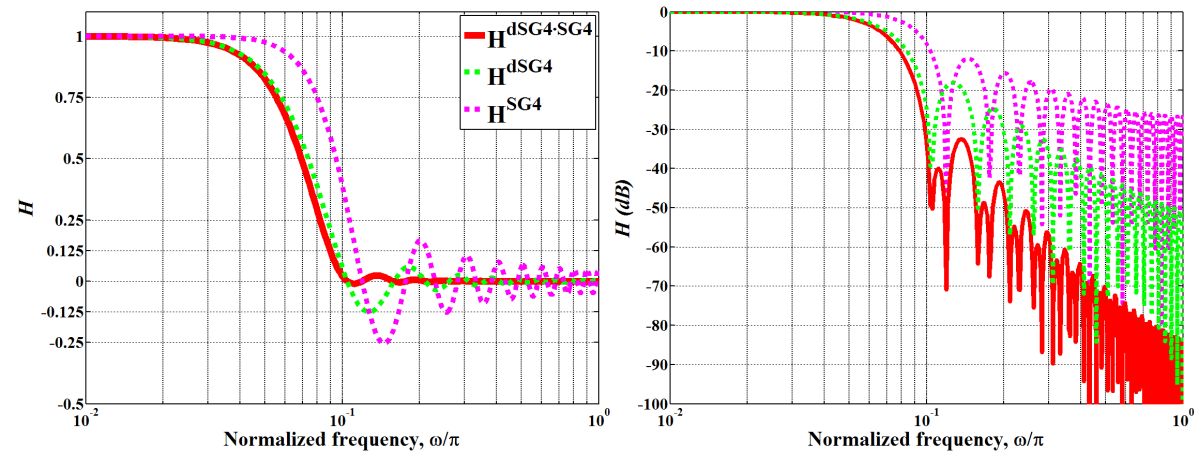

Figure B2. Left plot: example of the dSG4 - SG4 cascade (both with $N=20$ ) showing low-amplitude side lobes while preserving the passband extension of the dSG4 filter. $H \approx 1$ for $0<\omega / \pi<10^{-2}$. Right plot: same frequency responses plotted in decibel scale.

Operatively, one possible and efficient solution to compute "equivalent" cascade of two smoothing filters $(\mathrm{L} 1 \cdot \mathrm{L} 2)$ is to design the frequency response of the filter L2 to have a cutoff frequency, $\omega_{\mathrm{c}}$, large enough to include the pass band of L1 up to the beginning of its stop band (see Sect. 3.3). The following study shows an "equivalent" cascaded filter built from two SG filters. In this case, if the pass band must be preserved, it is useful to introduce the following relationship among $P, N$ and $\omega_{\mathrm{c}}$. For SG filters, the location of the cut-off frequency, $\omega_{\mathrm{c}}$, taken at $H_{-3 \mathrm{~dB}}$ (i.e., at $\left.\omega_{\mathrm{c}}=\omega(H \approx 0.7)\right)$ can be written (Schafer, 2011) as

$\omega_{\mathrm{c}}=\pi \frac{P+1}{3.2 N-4.6}, P=0,2,4 \cdots, N \geq 25, P<N$.

By using Eq. (B2) it can be seen that good results for an "equivalent" cascaded filter are obtained with the simple rule of using two $\mathrm{SG}$ filters with the same $N$ and $\Delta P=2$. As a consequence, in the resulting cascade filter, the stop band is much less affected by the presence of side lobes, and its pass band is nearly the same of the SG filter with the lower polynomial order (the "single" filter), as can be seen in Fig. B1. Moreover the simple rule applies also for $N<25$, i.e., beyond the validity limits of Eq. (B2).

However, the transient zone of the cascade filter increases; this means that if $N$ is the same in the two considered smoothing filters, the loss of information at the boundaries of the output signal is doubled when compared to the case with a single filter application. Efficient "equivalent filters" are obtained cascading an SG filter and a dSG filter with the same $P$ and $N$, as shown in Fig. B2. Anyhow the difference in pass band between the cascaded and the "single" filter is slightly more pronounced than in the previous case. The lat- 
ter "equivalent" cascaded filter shows side lobes with amplitude close to 0.02 , i.e., almost negligible for practical purposes. Moreover, it is also computationally efficient because both the needed impulse and frequency responses are calculated simultaneously by the SG algorithm (see Appendix A).

To summarize, operating with such cascaded filters retains all the advantages of the SG filters with an added value in terms of efficiency for the high-frequency damping without introducing high-frequency artifacts, though the transient zone increase could be a potential problem for lidar applications. If not otherwise stated, the value of $N$ associated with a cascade of two smoothing filters is the same for both filters.

\section{Appendix C: Windowed filters}
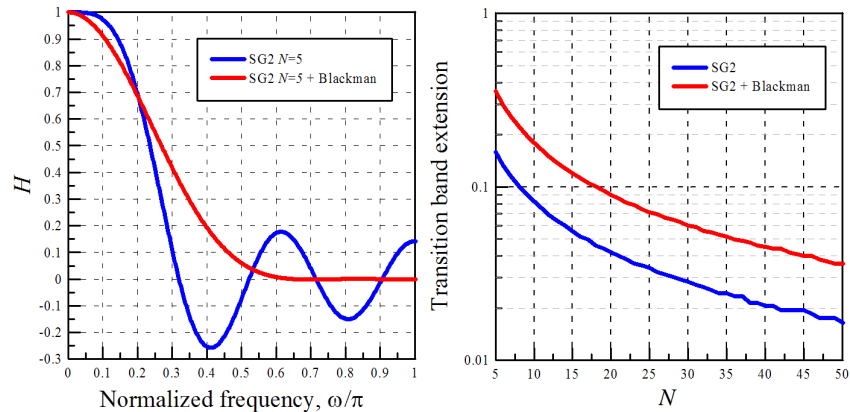

Figure C1. Left panel: frequency response of an SG2 filter with $N=5$, which exhibits unwanted side lobes (blue line). This problem is practically removed using a Blackman-type window. The resulting SG2-windowed filter frequency response (red line) shows an efficient suppression of the high frequencies, but it has an enlarged transition band (about a factor of 2 , for any $N$ ).

The impulse response of the ideal low-pass filter is of infinite length (Mitra, 2001); for the FIR design it must be truncated. This could be done by the multiplication of the impulse response by a rectangular window function (Mitra, 2001). However, the frequency response $H(\omega)$ calculated by Eq. (3) with the impulse response truncated in this way will exhibit unwanted oscillations in both its pass band and stop band, i.e., side lobes: the Gibbs phenomenon (Mitra, 2001). In order to reduce this phenomenon several types of window functions are reported in literature (Harris, 1978). However, each window function involves a trade-off between the requirements of high spectral selectivity (i.e., a narrow transition band) and strong suppression of noise (low side lobes) (Mitra, 2001). Eisele has successfully introduced a Blackman-type window function in lidar signal's analysis (Eisele, 1998; Trickl, 2010):

$$
\begin{aligned}
& w^{\mathrm{Blk}}(k) \\
& =\left\{\begin{array}{l}
0.42+0.5 \cos \left(\pi \frac{k}{N}\right)+0.08 \cos \left(2 \pi \frac{k}{N}\right),-N \leq k \leq N \\
0, \text { elsewhere. }
\end{array}\right.
\end{aligned}
$$

As an example of the application of the window method, a Blackman-type window like the one shown in Eq. (C1) has been applied to an SG2 impulse response. The result in Fig. $\mathrm{C} 1$ shows a frequency response with very small side lobes (maximum amplitude $\sim 0.001$ ); see also Fig. D1 (lower panel). It is also evident that using the same $N$, the pass-band extension of the SG2-windowed filter is nearly the same as the SG2.

As shown in the right plot of Fig. C1, the transition band, defined as $\left(\left.\omega\right|_{H=0.1}-\left.\omega\right|_{H=0.9}\right) / \pi$ (Cappellini and Emiliani, 1986), in the SG2-windowed filter, is larger (about a factor of 2) than the one of SG2 filter (left plot in Fig. C1). The SG2windowed filter, in Fig. $\mathrm{C} 1$, shows a worse signal preservation in the pass band (i.e., for $\omega / \pi<0.2$ ) with respect to the SG2 filter.

\section{Appendix D: The Gaussian filter}

The Gaussian filter $(G)$ is widely adopted to smooth signals especially in image processing (ter Haar Romeny, 2003). This filter is characterized by a single parameter $(\sigma$, the standard deviation), and its impulse response (a zero mean Gaussian) has the advantage that it can be written analytically as

$$
\begin{aligned}
& h_{\sigma}(k)=\left(2 \pi \sigma^{2}\right)^{-1 / 2} e^{-\frac{k^{2}}{2 \sigma^{2}}} ;-\infty \leq k \leq \infty \\
& h_{\sigma}^{(1) \mathrm{L}}(k)=\frac{d h_{\sigma}(k)}{\mathrm{d} k}=-\frac{k}{\sigma^{2}} h_{\sigma}(k) ;-\infty \leq k \leq \infty .
\end{aligned}
$$

In Eq. (D1), $h_{\sigma}(k)$ is the impulse response of the Gaussian filter and $h_{\sigma}^{(1) \mathrm{L}}(k)$ is that for the Gaussian first-derivative filter. The convolution of a signal with a Gaussian firstderivative filter is equivalent to differentiating the signal before (or after) the low-pass filtering operation. This means that, as seen for SG derivative filters, the Gaussian derivative filter also has a low-pass filtering behavior inherently embedded. Moreover, the frequency response of the low-pass filter embedded in the Gaussian first-derivative filter is a Gaussian filter with the same parameter $\sigma$ (Hale, 2011). The Fourier transform of a Gaussian function is everywhere non-zero and, therefore, cannot be sampled without some aliasing. The aliasing will become negligible if $\sigma \geq 1$ (Hale, 2011), although even a slightly lower value is allowed by some authors (ter Haar Romeny, 2003). The impulse responses in Eq. (D1) must be truncated. However, the Gaussian curve has a very strong decay to zero and for this reason it can be truncated without appreciable effects: for $|k| \geq 4 \sigma, h_{\sigma}(k)$ is $<0.4 \%$ of its maximum value (Hale, 2011; Young and van Vliet, 1995), while $\left|h_{\sigma}^{(1) \mathrm{L}}(k)\right|$ is $<3 \%$. These latter conditions imply that in order to properly truncate the impulse responses it is sufficient to set $N$ equal to $4 \sigma$ (actually the nearest integer to $4 \sigma$ ) in Eq. (2).

When $\sigma$ increases, the pass band reduces its extension and provides a stronger smoothing effect, although a Gaussian filter has a transition band quite wider than the SG filter with 

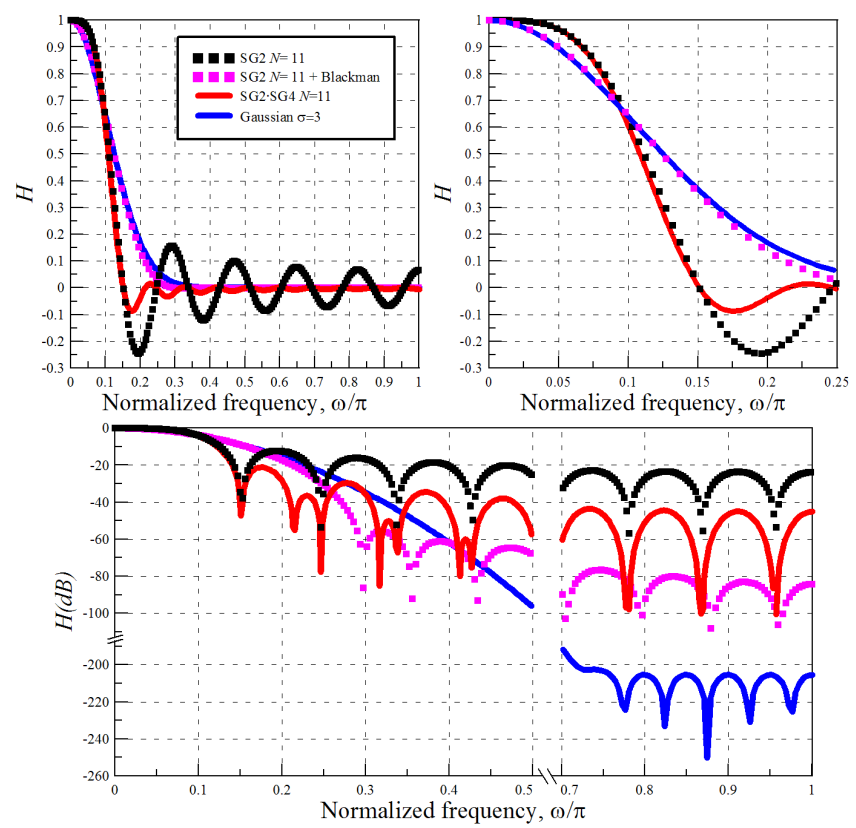

Figure D1. Comparison among filters designed with parameters making their pass bands similar. The upper-left panel shows the differences in the stop-band regions, due to the presence of side lobes; the SG2 filter (black squares line) has the worst behavior. In the upper-right panel there is a close-up view of the upper-left plot, and the different behavior in the pass bands and transition bands is quite evident. The cascaded SG2 -SG4 and the SG2 filters have the best performances. In the bottom plot, the same frequency responses of the upper plots are reported in decibel scale.

similar pass band. A Gaussian filter is also less flat in the pass band (van Vliet et al., 1998) than an SG filter with $P \geq 2$, but it has the advantage of having almost no side lobes.

Figure D1 summarizes the performances of the low-pass filters described so far in Appendix A-C. It can be seen that the Gaussian filter exhibits a behavior quite similar to the SG2 with a Blackman-type window and that they both have very small side lobes (visible only in the lower panel of Fig. D1, decibel scale). Figure D1 also shows that the SG2 has a better behavior in the pass band $(\omega / \pi<0.08)$ than Gaussian/SG2-windowed filters, i.e., less distortion in the pass band though it is heavily affected by side lobes. Both the Gaussian and the SG2-windowed filters also exhibit a much slower transition to the stop band as compared to the others. Finally, the cascaded filter shows good pass-band behavior and reduced side lobes but at the expense of a larger transient. 
Acknowledgements. The financial support for EARLINET in the ACTRIS Research Infrastructure Project by the European Union's Horizon 2020 research and innovation programme under grant agreement no. 654169 and previously under grant agreement no. 262254 in the 7th Framework Programme (FP7/2007-2013) is gratefully acknowledged.

Edited by: A. Ansmann

\section{References}

Ansmann, A., Wandinger, U., Riebesell, M., Weitkamp, C., and Michaelis, W.: Independent measurement of extinction and backscatter profiles in cirrus clouds by using a combined Raman elastic-backscatter lidar, Appl. Optics, 31, 7113-7131, 1992.

Barak, P.: Smoothing and Differentiation by an Adaptive-Degree Polynomial Filter, Anal. Chem., 67, 2758-2762, 1995.

Beyerle, G. and McDermid, I. S.: Altitude range resolution of differential absorption lidar ozone profiles, Appl. Optics, 38, 924-927, 1999.

Born, M. and Wolf, E.: Principles of Optics, 7th Edition, Cambridge University Press, Cambridge, United Kingdom, 985 pp., 1999.

Bromba, M. U. A. and Ziegler, H.: Application hints for SavitzkyGolay digital smoothing filters, Anal. Chem., 53, 1583-1586, 1981.

Brown, C. D., Vega-Montoto, L., and Wentzell, P. D.: Derivative Preprocessing and Optimal Corrections for Baseline Drift in Multivariate Calibration, Appl. Spectrosc., 54, 1055-1068, 2000.

Cappellini V. and Emiliani P. L.: Two-Dimensional Digital Systems and Applications: the State of the Art, in: Multidimensional System - Technique and Applications, edited by: Tzafestas, S. G., Marcel Dekker Inc., New York, United States of America, 1-35, 1986.

D’Amico, G., Amodeo, A., Baars, H., Binietoglou, I., Freudenthaler, V., Mattis, I., Wandinger, U., and Pappalardo, G.: EARLINET Single Calculus Chain - overview on methodology and strategy, Atmos. Meas. Tech., 8, 4891-4916, doi:10.5194/amt-84891-2015, 2015.

D'Antona, G. and Ferrero, A.: Digital Signal Processing for Measurement Systems - Theory and Applications, Springer Science+Business Media, Inc., New York, United States of America, 268 pp., 2006.

Das, S. and Chakraborty M.: QRS Detection Algorithm Using Savitzky-Golay Filter, ACEEE Int. J. on Signal \& Image Processing, 3, 55-58, 2012.

Eisele, H.: Aufbau und Betrieb eines Dreiwellenlängen-Lidars für Ozonmessungen in der gesamten Troposphäre und Entwicklung eines neuen Auswerteverfahrens zur Aerosolkorrektur, Dissertation, Universität Tübingen (1997), Schriftenreihe des Fraunhofer-Instituts für Atmosphärische Umweltforschung, 55, Wissenschafts-Verlag Maraun, Frankfurt/Main, Germany, 107 pp., 1998 (in German).

Eisele, H. and Trickl, T.: Improvements of the aerosol algorithm in ozone-lidar data processing by use of evolutionary strategies, Appl. Opt., 44, 2638-2651, 2005.

Enke, C. G. and Nieman T. A.: Signal-to-noise ratio enhancement by least-squares polynomial smoothing, Anal. Chem. 48, 705A712A, 1976.
Fernald, F. G.: Analysis of atmospheric lidar observations: some comments, Appl. Opt., 23, 652-653, 1984.

Gans, P.: Data Fitting in the Chemical Sciences: By the Method of Least Squares, Wiley \& Sons Ltd, Chichester, England, 270 pp., 1992.

Gans, P. and Gill, J. B.: Examination of the Convolution Method for Numerical Smoothing and Differentiation of Spectroscopic Data in Theory and in Practice, Appl. Spectrosc. 37, 515-520, 1983.

Godin S.: Étude expérimentale par télédétection laser et modélisation del la distribution verticale d'ozone dans la hute stratospherè, $\mathrm{PhD}$ thesis, Univerisité Pierre et Marie Curie, Paris, 231 pp., 1987.

Godin, S., Carswell, A. I., Donovan, D. P., Claude, H., Steinbrecht, W., McDermid, I. S., McGee, T. J., Gross, M. R., Nakane, H., Swart, D. P. J., Bergwerff, H. B., Uchino, O., von der Gathen, P., and Neuber, R.: Ozone differential absorption lidar algorithm intercomparison, Appl. Optics, 38, 6225-6236, 1999.

Gorry, P.: General least-squares smoothing and differentiation by the convolution (Savitzky-Golay) method, Anal. Chem., 62, 570573, 1990.

Hale D.: Recursive Gaussian filters, Center for wave phenomena/Colorado School of Mines, CWP-546, available at: http: //www.cwp.mines.edu/Meetings/Project06/cwp546.pdf (last access: 14 May 2012), 2011.

Hamming, R. W.: Digital filters, Third Edition, Dover Publications Inc., Mineola (NY), United States of America, 284 pp., 1998.

Harris, F. J.: On the Use of Windows for Harmonic Analysis with the Discrete Fourier Transform, Proceedings of the IEEE, 66, 5183, 1978.

Karam, L. J., McClellan, J. H., Selesnick, I. W., and Burrus, C. S.: Digital Filtering, in: The Digital Signal Processing Handbook Digital Signal Processing Fundamentals, Second Edition, edited by: Madisetti, V. K., CRC Press, Boca Raton (FL), United States of America, 11-1-11-89, 2009.

Khan, A.: Problems of Smoothing and Differentiation of Data by Least-Squares Procedures and Possible Solutions, Anal. Chem., 59, 654-657, 1987.

Klett, J. D.: Stable analytic inversion solution for processing lidar returns, Appl. Opt., 20, 211-220, 1981.

Kohn, A. F.: Phase distortion in biological signal analysis caused by linear phase FIR filters, Med. Biol. Eng. Comput., 25, 231-238, 1987.

Kuc, R.: Introduction to Digital Signal Processing, McGraw-Hill Book Co., Singapore, 474 pp, 1988.

Leach, R. A., Carter, C. A., and Harris, J. M.: Least-squares polynomial filters for initial point and slope estimation, Anal. Chem., 56, 2304-2307, 1984.

Leblanc, T., Godin-Beekmann, S., Payen, G., Gabarrot, F., Van Gijsel, A., Bandoro, J., Sica, R., and Trickl, T.: Standardization of the definitions of vertical resolution and uncertainty in the NDACC-archived ozone and temperature lidar measurements, in: Reviewed \& Revised Papers 26th International Laser Radar Conference, Vol. 1, Porto Heli, Greece, 25-29 June 2012, 327330, 2012.

Luo, J., Ying, K., He, P., and Bai J.: Properties of Savitzky-Golay digital differentiators, Digital Signal Processing, 15, 122-136, 2005 . 
Masci, F.: Algorithms for the inversion of lidar signals: RayleighMie measurements in the stratosphere, Annali di Geofisica, 42 , 71-83, 1999.

Matthias, V., Balis, D., Bösenberg, J., Eixmann, R., Iarlori, M., Komguem, L., Mattis, I., Papayannis, A., Pappalardo, G., Perrone, M. R., and Wang, X.: Vertical aerosol distribution over Europe: Statistical analysis of Raman lidar data from 10 European Aerosol Research Lidar Network (EARLINET) stations, J. Geophys. Res.-Atmos, 109, D18201, doi:10.1029/2004JD004638, 2004.

McClellan, J., Schafer, R., and Yoder M. A.: Signal Processing First, Prentice Hall, Pearson Education, Inc., Upper Saddle River, NJ, 489 pp., 2003.

McGee, T. J., Gross, M. R., Butler, J. J., and Kimvilakani, P. E.: Improved stratospheric ozone LIDAR, Opt. Eng., 34, 1421-1430, 1995.

Mitra, S.: Digital Signal Processing 2nd Ed, McGraw-Hill, New York, 866 pp., 2001.

Mollova, G.: Compact formulas for least-squares design of digital differentiators, Electron. Lett., 35, 1695-1697, 1999.

Oppenheim, A. V. and Schafer R. W.: Discrete-Time Signal Processing, 3ed, Prentice Hall, Upper Saddle River, NJ, 1120 pp., 2009.

Orfanidis, S. J.: Introduction to Signal Processing, available at: http: //eceweb1.rutgers.edu/ orfanidi/intro2sp/, (last access: 13 December 2011), 2010.

Pappalardo, G., Amodeo, A., Pandolfi, M., Wandinger, U., Ansmann, A., Bosenberg, J., Matthias, V., Amiridis, V., De Tomasi, F., Frioud, M., Iarlori, M., Komguem, L., Papayannis, A., Rocadenbosch, F., and Wang, X.: Aerosol lidar intercomparison in the framework of the EARLINET project. 3. Raman lidar algorithm for aerosol extinction, backscatter and lidar ratio, Appl. Optics, 43, 5370-5385, 2004.

Pornsawad, P., D’Amico, G., Böckmann, C., Amodeo, A., and Pappalardo, G.: Retrieval of aerosol extinction coefficient profiles from Raman lidar data by inversion method, Appl. Optics, 51, 2035-2044, 2012.

Press, W. H., Teukolsky, S, A., Vetterling, W. T., and Flannery, B. P.: Numerical Recipes 3rd Edition: The Art of Scientific Computing, 3 ed., Cambridge University Press, New York, NY, USA, 1256 pp., 2007.

Rabiner L. R. and Gold B.: Theory and Applications of Digital Signal processing, Prentice-Hall, Englewood Cliffs (NY), United States of America, 777 pp., 1975.

Rabiner, L. R., Gold, B., and McGonegal, C. A.: An approach to the approximation problem for nonrecursive digital filters, IEEE Transactions on Audio and Electroacoustics, AU-18, 83-106, 1970.

Rocadenbosch, F., Frasier S., Kumar D., Lange D., Gregorio E., and Sicard M.: Backscatter error bounds for the elastic lidar twocomponent inversion algorithm, IEEE Trans. Geosc. Rem. Sensing, 50, 4791-4803, 2012.

Rzhevskii, A. M. and Mardilovich, P. P.: Generalized Gans-Gill Method for Smoothing and Differentiation of Composite Profiles in Practice, Appl. Spectrosc., 48, 13-20, 1994.

Savitzky, A. and Golay M. J. E.: Smoothing and differentiation of data by simplified least squares procedures, Anal. Chem., 36, 1627-1639, 1964.
Schafer, R. W.: What is a Savitzky-Golay filter?, IEEE Signal Process. Mag., 28, 111-117, 2011.

Shcherbakov, V.: Regularized algorithm for Raman lidar data processing, Appl. optics, 46, 4879-4889, 2007.

Smith, J. O. III: Introduction to Digital Filters: with Audio Applications, available at: http://ccrma.stanford.edu/ jos/filters/ (last access: 12 December 2014), 2007.

Steffen, P.: On digital smoothing filters: A brief review of closed form solutions and two new filter approaches, Circ. Syst. Signal Pr., 5, 187-210, 1986.

ter Haar Romeny, B. M.: Front-end vision and multi-scale image analysis: multi-scale computer vision theory and applications, written in Mathematica, Series: Computational Imaging and Vision, Vol. 27, edited by: Viergever, M., A., Kluwer Academic Publishers, Dordrecht, the Netherlands, 466 pp., 2003.

Trickl, T.: Tropospheric trace-gas measurements with the differential-absorption lidar technique, in: Recent Advances in Atmospheric Lidars, edited by: Fiorani, L., Mitev, V., INOE Publishing House, Bucharest, Romania, 2010, Series on Optoelectronic Materials and Devices, 7, 87-147, 2010.

Turton, B. C. H.: A novel variant of the Savitzky-Golay filter for spectroscopic applications, Meas. Sci. Technol., 3, 858-863, 1992.

van Vliet, L. J., Young, I. T., and Verbeek, P. W.: Recursive gaussian derivative filters in: Proceedings of the 14th International Conference on Pattern Recognition, Vol. I, Brisbane, Australia, 16-20 August 1998, 509-514, 1998.

VDI: Remote sensing - Atmospheric measurements with LIDAR - Measuring gaseous air pollution with DAS LIDAR, Verein Deutscher Ingenieure, Düsseldorf, Germany, Guideline VDI 4210, Part 1, 47 pp., 1999.

Vogelmann, H. and Trickl, T.: Wide-Range Sounding of FreeTropospheric Water Vapor with a Differential-Absorption Lidar (DIAL) at a High-Altitude Station, Appl. Opt., 47, 21162132, 2008.

Wandinger, U. and Ansmann A.: Experimental determination of the lidar overlap profile with Raman lidar, Appl. Optics, 41, 511514, 2002.

Wang, Y.: An Effective Approach to Finding Differentiator Window Functions Based on Sinc Sum Function, Circ. Syst. Signal Pr., 31, 1809-1828, 2012.

Wang, Y.: New Window Functions for the Design of Narrowband Lowpass Differentiators, Circ. Syst. Signal Pr., 32, 1771-1790, 2013.

Whiteman, D. N.: Application of statistical methods to the determination of slope in lidar data, Appl. Optics, 38, 3360-3369, 1999.

Wulfmeyer, V. and Bösenberg, J.: Ground-based differential absorption lidar for water-vapor profiling: assessment of accuracy, resolution, and meteorological applications, Appl. Optics, 37, 38253844, 1998.

Young, I. T. and Van Vliet, L. J.: Recursive implementation of the Gaussian filter, Signal Process., 44, 139-151, 1995.

Ziegler, H.: Properties of Digital Smoothing Polynomial (DISPO) Filters, Appl. Spectrosc., 35, 88-92, 1981.

Zuo, C., Chen, Q., Yu, Y., and Asundi A.: Transport-of-intensity phase imaging using Savitzky-Golay differentiation filter - theory and applications, Opt. Express, 21, 5346-5362, 2013. 